\title{
Strong decays of the $Y(4660)$ as a vector tetraquark state in solid quark-hadron duality
}

\author{
Zhi-Gang Wang ${ }^{\mathrm{a}}$ \\ Department of Physics, North China Electric Power University, Baoding 071003, People's Republic of China
}

Received: 14 January 2019 / Accepted: 19 February 2019 / Published online: 1 March 2019

(C) The Author(s) 2019

\begin{abstract}
In this article, we choose the $[s c]_{P}[\bar{s} \bar{c}]_{A}-$ $[s c]_{A}[\bar{s} \bar{c}]_{P}$ type tetraquark current to study the hadronic coupling constants in the strong decays $Y(4660) \rightarrow J / \psi f_{0}$ (980), $\eta_{c} \phi(1020), \chi_{c 0} \phi(1020), D_{s} \bar{D}_{s}, D_{s}^{*} \bar{D}_{s}^{*}, D_{s} \bar{D}_{s}^{*}, D_{s}^{*} \bar{D}_{s}$, $\psi^{\prime} \pi^{+} \pi^{-}, J / \psi \phi(1020)$ with the QCD sum rules based on solid quark-hadron quality. The predicted width $\Gamma(Y(4660))$ $=74.2_{-19.2}^{+29.2} \mathrm{MeV}$ is in excellent agreement with the experimental data $68 \pm 11 \pm 1 \mathrm{MeV}$ from the Belle collaboration, which supports assigning the $Y(4660)$ to be the $[s c]_{P}[\bar{s} \bar{c}]_{A}-$ $[s c]_{A}[\bar{s} \bar{c}]_{P}$ type tetraquark state with $J^{P C}=1^{--}$. In calculations, we observe that the hadronic coupling constants $\left|G_{Y \psi^{\prime} f_{0}}\right| \gg\left|G_{Y J / \psi f_{0}}\right|$, which is consistent with the observation of the $Y(4660)$ in the $\psi^{\prime} \pi^{+} \pi^{-}$mass spectrum, and favors the $\psi^{\prime} f_{0}(980)$ molecule assignment. It is important to search for the process $Y(4660) \rightarrow J / \psi \phi(1020)$ to diagnose the nature of the $Y(4660)$, as the decay is greatly suppressed.
\end{abstract}

\section{Introduction}

In 2007, the Belle collaboration observed the $Y(4360)$ and $Y(4660)$ in the $\pi^{+} \pi^{-} \psi^{\prime}$ invariant mass distribution with statistical significances $8.0 \sigma$ and $5.8 \sigma$ respectively in the precess $e^{+} e^{-} \rightarrow \gamma_{\mathrm{ISR}} \pi^{+} \pi^{-} \psi^{\prime}$ between threshold and $\sqrt{s}=5.5 \mathrm{GeV}$ using $673 \mathrm{fb}^{-1}$ of data collected with the Belle detector at KEKB [1]. In 2008, the Belle collaboration observed the $Y$ (4630) in the $\Lambda_{c}^{+} \Lambda_{c}^{-}$invariant mass distribution with a significance of $8.2 \sigma$ in the exclusive process $e^{+} e^{-} \rightarrow \gamma_{\mathrm{ISR}} \Lambda_{c}^{+} \Lambda_{c}^{-}$with an integrated luminosity of $695 \mathrm{fb}^{-1}$ at the KEKB [2]. The values of the mass and width of the $Y(4630)$ are consistent within errors with that of the new charmonium-like state $Y(4660)$.

In 2014, the Belle collaboration measured the $e^{+} e^{-} \rightarrow$ $\gamma_{\text {ISR }} \pi^{+} \pi^{-} \psi^{\prime}$ cross section from 4.0 to $5.5 \mathrm{GeV}$ with the full data sample of the Belle experiment using the ISR (initial state radiation) technique, and determined the parameters of

a e-mail: zgwang@aliyun.com the $Y(4360)$ and $Y(4660)$ resonances and superseded previous Belle determination [3]. The masses and widths are shown explicitly in Table 1. Furthermore, the Belle collaboration studied the $\pi^{+} \pi^{-}$invariant mass distribution and observed that there are two clusters of events around the masses of the $f_{0}(500)$ and $f_{0}(980)$ corresponding to the $Y(4360)$ and $Y(4660)$, respectively. The $J^{P C}$ quantum numbers of the final states accompanying the ISR photon(s) are restricted to $J^{P C}=1^{--}$. According to potential model calculations [4-6], the $4^{3} \mathrm{~S}_{1}, 5^{3} \mathrm{~S}_{1}, 6^{3} \mathrm{~S}_{1}$ and $3^{3} \mathrm{D}_{1}$ charmonium states are expected to be in the mass range close to the two resonances $Y$ (4360) and $Y(4660)$, however, there are no enough vector charmonium candidates which can match those new $Y$ states consistently.

Now, let us begin with discussing the nature of the $f_{0}(500)$ and $f_{0}(980)$ to explore the $Y(4660)$. In the scenario of conventional two-quark states, the structures of the $f_{0}(500)$ and $f_{0}(980)$ in the ideal mixing limit can be symbolically written as,

$f_{0}(500)=\frac{\bar{u} u+\bar{d} d}{\sqrt{2}}, f_{0}(980)=\bar{s} s$.

While in the scenario of tetraquark states, the structures of the $f_{0}(500)$ and $f_{0}(980)$ in the ideal mixing limit can be symbolically written as [7-9],

$f_{0}(500)=u d \bar{u} \bar{d}, f_{0}(980)=\frac{u s \bar{u} \bar{s}+d s \bar{d} \bar{s}}{\sqrt{2}}$.

In Ref. [10], we take the nonet scalar mesons below $1 \mathrm{GeV}$ as the two-quark-tetraquark mixed states and study their masses and pole residues with the QCD sum rules in details. We determine the mixing angles, which indicate that the dominant components are the two-quark components. The $Y(4660)$ maybe have $\bar{s} s$ constituent. The decay $Y(4630) \rightarrow$ $\Lambda_{c}^{+} \Lambda_{c}^{-}$has been observed, if the $Y(4660)$ and $Y(4630)$ are the same particle, the decay $Y(4630) \rightarrow \Lambda_{c}^{+} \Lambda_{c}^{-}$is OkuboZweig-Iizuka suppressed, there should be some rescattering mechanism to account for the decay. 
Table 1 The masses and widths from the different experiments

\begin{tabular}{llll}
\hline Year & Mass $(\mathrm{MeV})$ & Width $(\mathrm{MeV})$ & Experiment \\
\hline 2007 & & & \\
$Y(4360)$ & $4361 \pm 9 \pm 9$ & $74 \pm 15 \pm 10$ & Belle [1] \\
$Y(4660)$ & $4664 \pm 11 \pm 5$ & $48 \pm 15 \pm 3$ & Belle [1] \\
2008 & & & \\
$Y(4630)$ & $4634_{-7-8}^{+8+5}$ & $92_{-24-21}^{+40+10}$ & Belle [2] \\
2014 & & & \\
$Y(4360)$ & $4347 \pm 6 \pm 3$ & $103 \pm 9 \pm 5$ & Belle [3] \\
$Y(4660)$ & $4652 \pm 10 \pm 8$ & $68 \pm 11 \pm 1$ & Belle [3] \\
\hline
\end{tabular}

Table 2 The masses from the QCD sum rules with different quark structures, where the OPE denotes truncations of the operator product expansion up to the vacuum condensates of dimension $n$, the No denotes the vacuum condensates of dimension $n^{\prime}$ are not included

\begin{tabular}{lllll}
\hline & Structures & $\begin{array}{l}\text { OPE } \\
(\mathrm{No})\end{array}$ & $\begin{array}{l}\text { Mass } \\
(\mathrm{GeV})\end{array}$ & References \\
\hline$Y(4660)$ & $\psi^{\prime} f_{0}(980)$ & 10 & 4.71 & {$[14]$} \\
$Y(4660)$ & $\psi^{\prime} f_{0}(980)$ & 6 & 4.67 & {$[15]$} \\
$Y(4660)$ & {$[s c]_{S}[\bar{s} \bar{c}]_{V}+[s c]_{V}[\bar{s} \bar{c}]_{S}$} & $8(7)$ & 4.65 & {$[20]$} \\
$Y(4660)$ & {$[s c]_{S}[\bar{s} \bar{c}]_{V}+[s c]_{V}[\bar{s} \bar{c}]_{S}$} & 10 & 4.68 & {$[21]$} \\
$Y(4660)$ & {$[q c]_{S}[\bar{q} \bar{c}]_{V}+[q c]_{V}[\bar{q} \bar{c}]_{S}$} & $8(7)$ & 4.64 & {$[22]$} \\
$Y(4360)$ & {$[q c]_{S}[\bar{q} \bar{c}]_{V}+[q c]_{V}[\bar{q} \bar{c}]_{S}$} & 10 & 4.34 & {$[24]$} \\
$Y(4660)$ & {$[s c]_{P}[\bar{s} \bar{c}]_{A}-[s c]_{A}[\bar{s} \bar{c}]_{P}$} & 10 & 4.70 & {$[23]$} \\
$Y(4660)$ & {$[s c]_{P}[\bar{s} \bar{c}]_{A}-[s c]_{A}[\bar{s} \bar{c}]_{P}$} & 10 & 4.66 & {$[24]$} \\
$Y(4660)$ & {$[q c]_{P}[\bar{q} \bar{c}]_{A}-[q c]_{A}[\bar{q} \bar{c}]_{P}$} & 10 & 4.66 & {$[23]$} \\
$Y(4660)$ & {$[q c]_{P}[\bar{q} \bar{c}]_{A}-[q c]_{A}[\bar{q} \bar{c}]_{P}$} & 10 & 4.59 & {$[24]$} \\
$Y(4660)$ & {$[q c]_{A}[\bar{q} \bar{c}]_{A}$} & 10 & 4.66 & {$[25]$} \\
$Y(4660)$ & {$[s c]_{S}[\bar{s} \bar{c}]_{S}$} & 6 & 4.69 & {$[26]$} \\
\hline
\end{tabular}

The threshold of the $\psi^{\prime} f_{0}(980)$ is $4676 \mathrm{MeV}$ from the Particle Data Group [11], which is just above the mass $m_{Y(4660)}=4652 \pm 10 \pm 8 \mathrm{MeV}$ from the Belle collaboration [3]. The $Y(4660)$ can be assigned to be a $\psi^{\prime} f_{0}(980)$ molecular state [12-15] or a $\psi^{\prime} f_{0}(980)$ hadro-charmonium [16]. Other assignments, such as a $2 \mathrm{P}[c q]_{S}[\bar{c} \bar{q}]_{S}$ tetraquark state $[17,18]$, a $\psi(6 \mathrm{~S})$ state [6], a $\psi(5 \mathrm{~S})$ state [19], a ground state $\mathrm{P}$-wave tetraquark state [20-26] are also possible.

In Table 2, we list out the predictions of the masses of the vector tetraquark (tetraquark molecule) states based on the QCD sum rules $[14,15,20-26]$, where the $S, P, A$ and $V$ denote the scalar $(S)$, pseudoscalar $(P)$, axialvector $(A)$ and vector $(V)$ diquark states. From the Table, we can see that it is not difficult to reproduce the experimental value of the mass of the $Y(4660)$ with the QCD sum rules. However, the quantitative predications depend on the quark structures, the input parameters at the QCD side, the pole contributions of the ground states, and the truncations of the operator product expansion.
In the QCD sum rules for the hidden-charm (or hiddenbottom) tetraquark states and molecular states, the integrals

$\int_{4 m_{Q}^{2}(\mu)}^{s_{0}} d s \rho_{Q C D}(s, \mu) \exp \left(-\frac{s}{T^{2}}\right)$

are sensitive to the energy scales $\mu$, where the $\rho_{Q C D}(s, \mu)$ are the QCD spectral densities, the $T^{2}$ are the Borel parameters, the $s_{0}$ are the continuum thresholds parameters, the predicted masses depend heavily on the energy scales $\mu$. In Refs. [23,27-30], we suggest an energy scale formula $\mu=$ $\sqrt{M_{X / Y / Z}^{2}-\left(2 \mathbb{M}_{Q}\right)^{2}}$ with the effective $Q$-quark mass $\mathbb{M}_{Q}$ to determine the ideal energy scales of the QCD spectral densities. The formula enhances the pole contributions remarkably, we obtain the pole contributions as large as $(40-60) \%$, the largest pole contributions up to now. Compared to the old values obtained in Ref. [23], the new values based on detailed analysis with the updated parameters are preferred [24]. The energy scale formula also works well in the QCD sum rules for the hidden-charm pentaquark states [31-33].

For the correlation functions of the hidden-charm (or hidden-bottom) tetraquark currents, there are two heavy quark propagators and two light quark propagators, if each heavy quark line emits a gluon and each light quark line contributes a quark pair, we obtain a operator $G G \bar{q} q \bar{q} q$, which is of dimension 10, we should take into account the vacuum condensates at least up to dimension 10 in the operator product expansion.

In Refs. [23-25,34,35], we study the mass spectrum of the vector tetraquark states in a comprehensive way by carrying out the operator product expansion up to the vacuum condensates of dimension 10 , and use the energy scale formula $\mu=\sqrt{M_{X / Y / Z}^{2}-\left(2 \mathbb{M}_{c}\right)^{2}}$ or modified energy scale formula $\mu=\sqrt{M_{X / Y / Z}^{2}-\left(2 \mathbb{M}_{c}+0.5 \mathrm{GeV}\right)^{2}}=$ $\sqrt{M_{X / Y / Z}^{2}-(4.1 \mathrm{GeV})^{2}}$ to determine the ideal energy scales of the QCD spectral densities in a consistent way. In the scenario of tetraquark states, we observe that the preferred quark configurations for the $Y(4660)$ are the $[s c]_{P}[\bar{s} \bar{c}]_{A}-$ $[s c]_{A}[\bar{s} \bar{c}]_{P}$ and $[q c]_{A}[\bar{q} \bar{c}]_{A}$. In this article, we choose the quark configuration $[s c]_{P}[\bar{s} \bar{c}]_{A}-[s c]_{A}[\bar{s} \bar{c}]_{P}$ to examine the nature of the $Y(4660)$.

In Ref. [36], we assign the $Z_{c}^{ \pm}$(3900) to be the diquarkantidiquark type axialvector tetraquark state, study the hadronic coupling constants $G_{Z_{c} J / \psi \pi}, G_{Z_{c} \eta_{c} \rho}, G_{Z_{c} D \bar{D}^{*}}$ with the QCD sum rules by taking into account both the connected and disconnected Feynman diagrams in the operator product expansion. We pay special attentions to matching the hadron side of the correlation functions with the QCD side of the correlation functions to obtain solid duality. The routine works well in studying the decays $X(4140 / 4274) \rightarrow J / \psi \phi(1020)$ [37,38]. 
In this article, we assign the $Y(4660)$ to be the $[s c]_{P}[\bar{s} \bar{c}]_{A}-[s c]_{A}[\bar{s} \bar{c}]_{P}$ type vector tetraquark state, and study the strong decays $Y(4660) \rightarrow J / \psi f_{0}(980)$, $\eta_{c} \phi(1020), \chi_{c 0} \phi(1020), D_{s} \bar{D}_{s}, D_{s}^{*} \bar{D}_{s}^{*}, D_{s} \bar{D}_{s}^{*}, D_{s}^{*} \bar{D}_{s}$, $\psi^{\prime} \pi^{+} \pi^{-}, J / \psi \phi(1020)$ with the QCD sum rules based on the solid quark-hadron duality, and reexamine the assignment of the $Y(4660)$.

The article is arranged as follows: we illustrate how to calculate the hadronic coupling constants in the two-body strong decays of the tetraquark states with the QCD sum rules in Sect. 2, in Sect. 3, we obtain the QCD sum rules for the hadronic coupling constants $G_{Y J / \psi f_{0}}, G_{Y \eta_{c} \phi}, G_{Y \chi_{c 0} \phi}$, $G_{Y D_{s} \bar{D}_{s}}, G_{Y D_{s}^{*} \bar{D}_{s}^{*}}, G_{Y D_{s} \bar{D}_{s}^{*}}, G_{Y \psi^{\prime} f_{0}}, G_{Y J / \psi \phi}$; Sect. 4 is reserved for our conclusion.

\section{The hadronic coupling constants in the two-body strong decays of the tetraquark states}

In this section, we illustrate how to calculate the hadronic coupling constants in the two-body strong decays of the tetraquark states with the QCD sum rules. We write down the three-point correlation functions $\Pi(p, q)$ firstly,

$$
\Pi(p, q)=i^{2} \int d^{4} x d^{4} y e^{i p x} e^{i q y}\left\langle 0\left|T\left\{J_{B}(x) J_{C}(y) J_{A}^{\dagger}(0)\right\}\right| 0\right\rangle,
$$

where the currents $J_{A}(0)$ interpolate the tetraquark states $A$, the $J_{B}(x)$ and $J_{C}(y)$ interpolate the conventional mesons $B$ and $C$, respectively,

$$
\begin{aligned}
\left\langle 0\left|J_{A}(0)\right| A\left(p^{\prime}\right)\right\rangle & =\lambda_{A}, \\
\left\langle 0\left|J_{B}(0)\right| B(p)\right\rangle & =\lambda_{B}, \\
\left\langle 0\left|J_{C}(0)\right| C(q)\right\rangle & =\lambda_{C},
\end{aligned}
$$

the $\lambda_{A}, \lambda_{B}$ and $\lambda_{C}$ are the pole residues or decay constants.

At the phenomenological side, we insert a complete set of intermediate hadronic states with the same quantum numbers as the current operators $J_{A}(0), J_{B}(x), J_{C}(y)$ into the threepoint correlation functions $\Pi(p, q)$ and isolate the ground state contributions to obtain the result [39-41],

$$
\begin{aligned}
\Pi(p, q)= & \frac{\lambda_{A} \lambda_{B} \lambda_{C} G_{A B C}}{\left(m_{A}^{2}-p^{\prime 2}\right)\left(m_{B}^{2}-p^{2}\right)\left(m_{C}^{2}-q^{2}\right)} \\
& +\frac{1}{\left(m_{A}^{2}-p^{\prime 2}\right)\left(m_{B}^{2}-p^{2}\right)} \int_{s_{C}^{0}}^{\infty} d t \frac{\rho_{A C^{\prime}}\left(p^{\prime 2}, p^{2}, t\right)}{t-q^{2}} \\
& +\frac{1}{\left(m_{A}^{2}-p^{\prime 2}\right)\left(m_{C}^{2}-q^{2}\right)} \int_{s_{B}^{0}}^{\infty} d t \frac{\rho_{A B^{\prime}}\left(p^{\prime 2}, t, q^{2}\right)}{t-p^{2}} \\
& +\frac{1}{\left(m_{B}^{2}-p^{2}\right)\left(m_{C}^{2}-q^{2}\right)}
\end{aligned}
$$

$$
\begin{aligned}
& \times \int_{s_{A}^{0}}^{\infty} d t \frac{\rho_{A^{\prime} B}\left(t, p^{2}, q^{2}\right)+\rho_{A^{\prime} C}\left(t, p^{2}, q^{2}\right)}{t-p^{\prime 2}}+\cdots \\
= & \Pi\left(p^{\prime 2}, p^{2}, q^{2}\right),
\end{aligned}
$$

where $p^{\prime}=p+q$, the $G_{A B C}$ are the hadronic coupling constants defined by

$\left\langle B(p) C(q) \mid A\left(p^{\prime}\right)\right\rangle=i G_{A B C}$,

the four functions $\rho_{A C^{\prime}}\left(p^{\prime 2}, p^{2}, t\right), \quad \rho_{A B^{\prime}}\left(p^{\prime 2}, t, q^{2}\right)$, $\rho_{A^{\prime} B}\left(t^{\prime}, p^{2}, q^{2}\right)$ and $\rho_{A^{\prime} C}\left(t^{\prime}, p^{2}, q^{2}\right)$ have complex dependence on the transitions between the ground states and the higher resonances or the continuum states.

We rewrite the correlation functions $\Pi_{H}\left(p^{\prime 2}, p^{2}, q^{2}\right)$ at the hadron side as

$$
\begin{aligned}
& \Pi_{H}\left(p^{\prime 2}, p^{2}, q^{2}\right) \\
& =\int_{\left(m_{B}+m_{C}\right)^{2}}^{s_{A}^{0}} d s^{\prime} \int_{\Delta_{s}^{2}}^{s_{B}^{0}} d s \int_{\Delta_{u}^{2}}^{u_{C}^{0}} d u \frac{\rho_{H}\left(s^{\prime}, s, u\right)}{\left(s^{\prime}-p^{\prime 2}\right)\left(s-p^{2}\right)\left(u-q^{2}\right)} \\
& \quad+\int_{s_{A}^{0}}^{\infty} d s^{\prime} \int_{\Delta_{s}^{2}}^{s_{B}^{0}} d s \int_{\Delta_{u}^{2}}^{u_{C}^{0}} d u \frac{\rho_{H}\left(s^{\prime}, s, u\right)}{\left(s^{\prime}-p^{\prime 2}\right)\left(s-p^{2}\right)\left(u-q^{2}\right)}+\cdots,
\end{aligned}
$$

through dispersion relation, where the $\rho_{H}\left(s^{\prime}, s, u\right)$ are the hadronic spectral densities,

$$
\begin{aligned}
& \rho_{H}\left(s^{\prime}, s, u\right)=\lim _{\epsilon_{3} \rightarrow 0} \lim _{\epsilon_{2} \rightarrow 0} \lim _{\epsilon_{1} \rightarrow 0} \\
& \times \frac{\operatorname{Im}_{s^{\prime}} \operatorname{Im}_{s} \operatorname{Im}_{u} \Pi_{H}\left(s^{\prime}+i \epsilon_{3}, s+i \epsilon_{2}, u+i \epsilon_{1}\right)}{\pi^{3}},
\end{aligned}
$$

where the $\Delta_{s}^{2}$ and $\Delta_{u}^{2}$ are the thresholds, the $s_{A}^{0}, s_{B}^{0}, u_{C}^{0}$ are the continuum thresholds.

Now we carry out the operator product expansion at the QCD side, and write the correlation functions $\Pi_{Q C D}\left(p^{\prime 2}, p^{2}, q^{2}\right)$ as

$\Pi_{Q C D}\left(p^{\prime 2}, p^{2}, q^{2}\right)=\int_{\Delta_{s}^{2}}^{s_{B}^{0}} d s \int_{\Delta_{u}^{2}}^{u_{C}^{0}} d u \frac{\rho_{Q C D}\left(p^{\prime 2}, s, u\right)}{\left(s-p^{2}\right)\left(u-q^{2}\right)}+\cdots$,

through dispersion relation, where the $\rho_{Q C D}\left(p^{\prime 2}, s, u\right)$ are the QCD spectral densities,

$$
\begin{aligned}
\rho_{Q C D}\left(p^{\prime 2}, s, u\right)= & \lim _{\epsilon_{2} \rightarrow 0} \lim _{\epsilon_{1} \rightarrow 0} \\
& \times \frac{\operatorname{Im}_{s} \operatorname{Im}_{u} \Pi_{Q C D}\left(p^{\prime 2}, s+i \epsilon_{2}, u+i \epsilon_{1}\right)}{\pi^{2}} .
\end{aligned}
$$

However, the QCD spectral densities $\rho_{Q C D}\left(s^{\prime}, s, u\right)$ do not exist, 


$$
\begin{aligned}
& \rho_{Q C D}\left(s^{\prime}, s, u\right)=\lim _{\epsilon_{3} \rightarrow 0} \lim _{\epsilon_{2} \rightarrow 0} \lim _{\epsilon_{1} \rightarrow 0} \\
& \times \frac{\operatorname{Im}_{s^{\prime}} \operatorname{Im}_{s} \operatorname{Im}_{u} \Pi_{Q C D}\left(s^{\prime}+i \epsilon_{3}, s+i \epsilon_{2}, u+i \epsilon_{1}\right)}{\pi^{3}}=0,
\end{aligned}
$$

because

$\lim _{\epsilon_{3} \rightarrow 0} \frac{\operatorname{Im}_{s^{\prime}} \Pi_{Q C D}\left(s^{\prime}+i \epsilon_{3}, p^{2}, q^{2}\right)}{\pi}=0$.

Thereafter we will write the QCD spectral densities $\rho_{Q C D}\left(p^{\prime 2}, s, u\right)$ as $\rho_{Q C D}(s, u)$ for simplicity.

We math the hadron side of the correlation functions with the QCD side of the correlation functions, and carry out the integral over $d s^{\prime}$ firstly to obtain the solid duality [36],

$$
\begin{aligned}
& \int_{\Delta_{s}^{2}}^{s_{B}^{0}} d s \int_{\Delta_{u}^{2}}^{u_{C}^{0}} d u \frac{\rho_{Q C D}(s, u)}{\left(s-p^{2}\right)\left(u-q^{2}\right)} \\
& =\int_{\Delta_{s}^{2}}^{s_{B}^{0}} d s \int_{\Delta_{u}^{2}}^{u_{C}^{0}} d u \frac{1}{\left(s-p^{2}\right)\left(u-q^{2}\right)} \\
& \quad \times\left[\int_{\Delta^{2}}^{\infty} d s^{\prime} \frac{\rho_{H}\left(s^{\prime}, s, u\right)}{s^{\prime}-p^{\prime 2}}\right],
\end{aligned}
$$

the $\Delta^{2}$ denotes the thresholds $\left(m_{B}+m_{C}\right)^{2}$. Now we write down the quark-hadron duality explicitly,

$$
\begin{aligned}
& \int_{\Delta_{c}^{2}}^{s_{B}^{0}} d s \int_{\Delta_{u}^{2}}^{u_{C}^{0}} d u \frac{\rho_{Q C D}(s, u)}{\left(s-p^{2}\right)\left(u-q^{2}\right)} \\
& =\int_{\Delta_{c}^{2}}^{s_{B}^{0}} d s \int_{\Delta_{u}^{2}}^{u_{C}^{0}} d u \int_{\left(m_{B}+m_{C}\right)^{2}}^{\infty} d s^{\prime} \frac{\rho_{H}\left(s^{\prime}, s, u\right)}{\left(s^{\prime}-p^{\prime 2}\right)\left(s-p^{2}\right)\left(u-q^{2}\right)} \\
& =\frac{\lambda_{A} \lambda_{B} \lambda_{C} G_{A B C}}{\left(m_{A}^{2}-p^{\prime 2}\right)\left(m_{B}^{2}-p^{2}\right)\left(m_{C}^{2}-q^{2}\right)} \\
& +\frac{C_{A^{\prime} B}+C_{A^{\prime} C}}{\left(m_{B}^{2}-p^{2}\right)\left(m_{C}^{2}-q^{2}\right)} .
\end{aligned}
$$

No approximation is needed, we do not need the continuum threshold parameter $s_{A}^{0}$ in the $s^{\prime}$ channel. The $s^{\prime}$ channel and $s$ channel are quite different, we can not set the continuum threshold parameters in the $s$ channel as $s_{B}^{0}=s_{A}^{0}$, i.e. we can not set $s_{B}^{0}=s_{Y}^{0}=(5.15 \mathrm{GeV})^{2}$ in the present case, where the $B$ denotes the $J / \psi, \eta_{c}, \bar{D}_{s}, \bar{D}_{s}^{*}$, because the contaminations from the excited states $\psi^{\prime}, \eta_{c}^{\prime}, \bar{D}_{s}^{\prime}, \bar{D}_{s}^{* \prime}$ are out of control.

We can introduce the parameters $C_{A C^{\prime}}, C_{A B^{\prime}}, C_{A^{\prime} B}$ and $C_{A^{\prime} C}$ to parameterize the net effects,

$$
\begin{aligned}
C_{A C^{\prime}} & =\int_{s_{C}^{0}}^{\infty} d t \frac{\rho_{A C^{\prime}}\left(p^{\prime 2}, p^{2}, t\right)}{t-q^{2}}, \\
C_{A B^{\prime}} & =\int_{s_{B}^{0}}^{\infty} d t \frac{\rho_{A B^{\prime}}\left(p^{\prime 2}, t, q^{2}\right)}{t-p^{2}},
\end{aligned}
$$

$$
\begin{aligned}
C_{A^{\prime} B} & =\int_{s_{A}^{0}}^{\infty} d t \frac{\rho_{A^{\prime} B}\left(t, p^{2}, q^{2}\right)}{t-p^{\prime 2}}, \\
C_{A^{\prime} C} & =\int_{s_{A}^{0}}^{\infty} d t \frac{\rho_{A^{\prime} C}\left(t, p^{2}, q^{2}\right)}{t-p^{\prime 2}} .
\end{aligned}
$$

In numerical calculations, we take the relevant functions $C_{A^{\prime} B}$ and $C_{A^{\prime} C}$ as free parameters, and choose suitable values to eliminate the contaminations from the higher resonances and continuum states to obtain the stable QCD sum rules with the variations of the Borel parameters.

If the $B$ are charmonium or bottomnium states, we set $p^{\prime 2}=p^{2}$ and perform the double Borel transform with respect to the variables $P^{2}=-p^{2}$ and $Q^{2}=-q^{2}$, respectively to obtain the QCD sum rules,

$$
\begin{aligned}
& \frac{\lambda_{A} \lambda_{B} \lambda_{C} G_{A B C}}{m_{A}^{2}-m_{B}^{2}}\left[\exp \left(-\frac{m_{B}^{2}}{T_{1}^{2}}\right)\right. \\
& \left.\quad-\exp \left(-\frac{m_{A}^{2}}{T_{1}^{2}}\right)\right] \exp \left(-\frac{m_{C}^{2}}{T_{2}^{2}}\right) \\
& \quad+\left(C_{A^{\prime} B}+C_{A^{\prime} C}\right) \exp \left(-\frac{m_{B}^{2}}{T_{1}^{2}}-\frac{m_{C}^{2}}{T_{2}^{2}}\right) \\
& =\int_{\Delta_{s}^{2}}^{s_{B}^{0}} d s \int_{\Delta_{u}^{2}}^{u_{C}^{0}} d u \rho_{Q C D}(s, u) \exp \left(-\frac{s}{T_{1}^{2}}-\frac{u}{T_{2}^{2}}\right),
\end{aligned}
$$

where the $T_{1}^{2}$ and $T_{2}^{2}$ are the Borel parameters. If the $B$ are open-charm or open-bottom mesons, we set $p^{\prime 2}=4 p^{2}$ and perform the double Borel transform with respect to the variables $P^{2}=-p^{2}$ and $Q^{2}=-q^{2}$, respectively to obtain the QCD sum rules,

$$
\begin{aligned}
& \frac{\lambda_{A} \lambda_{B} \lambda_{C} G_{A B C}}{4\left(\tilde{m}_{A}^{2}-m_{B}^{2}\right)}\left[\exp \left(-\frac{m_{B}^{2}}{T_{1}^{2}}\right)\right. \\
& \left.\quad-\exp \left(-\frac{\tilde{m}_{A}^{2}}{T_{1}^{2}}\right)\right] \exp \left(-\frac{m_{C}^{2}}{T_{2}^{2}}\right) \\
& \quad+\left(C_{A^{\prime} B}+C_{A^{\prime} C}\right) \exp \left(-\frac{m_{B}^{2}}{T_{1}^{2}}-\frac{m_{C}^{2}}{T_{2}^{2}}\right) \\
& =\int_{\Delta_{s}^{2}}^{s_{B}^{0}} d s \int_{\Delta_{u}^{2}}^{u_{C}^{0}} d u \rho_{Q C D}(s, u) \exp \left(-\frac{s}{T_{1}^{2}}-\frac{u}{T_{2}^{2}}\right),
\end{aligned}
$$

where $\widetilde{m}_{A}^{2}=\frac{m_{A}^{2}}{4}$.

\section{The width of the $Y(4660)$ as a vector tetraquark state}

Now we write down the three-point correlation functions for the strong decays $Y(4660) \rightarrow J / \psi f_{0}(980), \eta_{c} \phi(1020)$, $\chi_{c 0} \phi(1020), \quad D_{s} \bar{D}_{s}, \quad D_{s}^{*} \bar{D}_{s}^{*}, \quad D_{s} \bar{D}_{s}^{*}, \quad D_{s}^{*} \bar{D}_{s}, \quad \psi^{\prime} \pi^{+} \pi^{-}$, 
$J / \psi \phi(1020)$, respectively, and apply the method presented in previous section to obtain the QCD sum rules for the hadronic coupling constants $G_{Y J / \psi f_{0}}, G_{Y \eta_{c} \phi}, G_{Y \chi_{c 0} \phi}$, $G_{Y D_{s} \bar{D}_{s}}, G_{Y D_{s}^{*} \bar{D}_{s}^{*}}, G_{Y D_{s} \bar{D}_{s}^{*}}, G_{Y \psi^{\prime} f_{0}}, G_{Y J / \psi \phi}$.

For the two-body strong decays $Y(4660) \rightarrow J / \psi f_{0}(980)$, $\psi^{\prime} f_{0}(980)^{*}$, the correlation function is

$$
\begin{aligned}
& \Pi_{\mu \nu}(p, q) \\
& \quad=i^{2} \int d^{4} x d^{4} y e^{i p x} e^{i q y}\left\langle 0\left|T\left\{J_{J / \psi, \mu}(x) J_{f_{0}}(y) J_{v}^{\dagger}(0)\right\}\right| 0\right\rangle,
\end{aligned}
$$

where

$$
\begin{aligned}
J_{J / \psi, \mu}(x)= & \bar{c}(x) \gamma_{\mu} c(x) \\
J_{f_{0}}(y)= & \bar{s}(y) s(y) \\
J_{\nu}(0)= & \frac{\varepsilon^{i j k} \varepsilon^{i m n}}{\sqrt{2}}\left[s^{T j}(0) C c^{k}(0) \bar{s}^{m}(0) \gamma_{\nu} C \bar{c}^{T n}(0)\right. \\
& \left.-s^{T j}(0) C \gamma_{\nu} c^{k}(0) \bar{s}^{m}(0) C \bar{c}^{T n}(0)\right]
\end{aligned}
$$

For the two-body strong decay $Y(4660) \rightarrow \eta_{c} \phi(1020)$, the correlation function is

$$
\begin{aligned}
& \Pi_{\mu \nu}(p, q) \\
& \quad=i^{2} \int d^{4} x d^{4} y e^{i p x} e^{i q y}\left\langle 0\left|T\left\{J_{\eta_{c}}(x) J_{\phi, \mu}(y) J_{v}^{\dagger}(0)\right\}\right| 0\right\rangle,
\end{aligned}
$$

where

$$
\begin{aligned}
J_{\eta_{c}}(x) & =\bar{c}(x) i \gamma_{5} c(x), \\
J_{\phi, \mu}(y) & =\bar{s}(y) \gamma_{\mu} s(y) .
\end{aligned}
$$

For the two-body strong decay $Y(4660) \rightarrow \chi_{c 0} \phi(1020)$, the correlation function is

$$
\begin{aligned}
& \Pi_{\mu \nu}(p, q) \\
& \quad=i^{2} \int d^{4} x d^{4} y e^{i p x} e^{i q y}\left\langle 0\left|T\left\{J_{\chi_{c 0}}(x) J_{\phi, \mu}(y) J_{\nu}^{\dagger}(0)\right\}\right| 0\right\rangle,
\end{aligned}
$$

where

$$
J_{\chi_{c 0}}(x)=\bar{c}(x) c(x) \text {. }
$$

For the two-body strong decay $Y(4660) \rightarrow D_{s} \bar{D}_{s}$, the correlation function is

$$
\begin{aligned}
& \Pi_{v}(p, q) \\
& \quad=i^{2} \int d^{4} x d^{4} y e^{i p x} e^{i q y}\left\langle 0\left|T\left\{J_{D_{s}}^{\dagger}(x) J_{D_{s}}(y) J_{v}^{\dagger}(0)\right\}\right| 0\right\rangle,
\end{aligned}
$$

where

$$
J_{D_{s}}(y)=\bar{s}(y) i \gamma_{5} c(y)
$$

For the two-body strong decay $Y(4660) \rightarrow D_{s}^{*} \bar{D}_{s}^{*}$, the correlation function is

$$
\begin{aligned}
& \Pi_{\alpha \beta \nu}(p, q) \\
& \quad=i^{2} \int d^{4} x d^{4} y e^{i p x} e^{i q y}\left\langle 0\left|T\left\{J_{D_{s}^{*}, \alpha}^{\dagger}(x) J_{D_{s}^{*}, \beta}(y) J_{v}^{\dagger}(0)\right\}\right| 0\right\rangle,
\end{aligned}
$$

where

$J_{D_{s}^{*}, \beta}(y)=\bar{s}(y) \gamma_{\beta} c(y)$.

For the two-body strong decay $Y(4660) \rightarrow D_{s} \bar{D}_{s}^{*}$, the correlation function is

$$
\begin{aligned}
& \Pi_{\mu \nu}(p, q) \\
& \quad=i^{2} \int d^{4} x d^{4} y e^{i p x} e^{i q y}\left\langle 0\left|T\left\{J_{D_{s}^{*}, \mu}^{\dagger}(x) J_{D_{s}}(y) J_{v}^{\dagger}(0)\right\}\right| 0\right\rangle .
\end{aligned}
$$

For the two-body strong decay $Y(4660) \rightarrow J / \psi \phi(1020)$, the correlation function is

$$
\begin{aligned}
& \Pi_{\alpha \beta \nu}(p, q) \\
& =i^{2} \int d^{4} x d^{4} y e^{i p x} e^{i q y}\left\langle 0\left|T\left\{J_{J / \psi, \alpha}(x) J_{\phi, \beta}(y) J_{\nu}^{\dagger}(0)\right\}\right| 0\right\rangle .
\end{aligned}
$$

At the phenomenological side, we insert a complete set of intermediate hadronic states with the same quantum numbers as the current operators into the three-point correlation functions and isolate the ground state contributions to obtain the hadron representation [39-41].

For the decays $Y(4660) \rightarrow J / \psi f_{0}(980), \psi^{\prime} f_{0}(980)^{*}$, the correlation function can be written as

$$
\begin{aligned}
\Pi_{\mu \nu}(p, q)= & \frac{f_{J / \psi} m_{J / \psi} f_{f_{0}} m_{f_{0}} \lambda_{Y} G_{Y J / \psi f_{0}}}{\left(p^{\prime 2}-m_{Y}^{2}\right)\left(p^{2}-m_{J / \psi}^{2}\right)\left(q^{2}-m_{f_{0}}^{2}\right)} \\
& \times\left(-g_{\mu \alpha}+\frac{p_{\mu} p_{\alpha}}{p^{2}}\right)\left(-g_{\nu}{ }^{\alpha}+\frac{p_{\nu}^{\prime} p^{\prime \alpha}}{p^{\prime 2}}\right) \\
& +\frac{f_{\psi^{\prime}} m_{\psi^{\prime}} f_{f_{0}} m_{f_{0}} \lambda_{Y} G_{Y \psi^{\prime} f_{0}}}{\left(p^{\prime 2}-m_{Y}^{2}\right)\left(p^{2}-m_{\psi^{\prime}}^{2}\right)\left(q^{2}-m_{f_{0}}^{2}\right)} \\
& \times\left(-g_{\mu \alpha}+\frac{p_{\mu} p_{\alpha}}{p^{2}}\right)\left(-g_{\nu}{ }^{\alpha}+\frac{p_{\nu}^{\prime} p^{\prime \alpha}}{p^{\prime 2}}\right)+\cdots \\
= & \Pi\left(p^{\prime 2}, p^{2}, q^{2}\right) g_{\mu \nu}+\cdots .
\end{aligned}
$$


For the decay $Y(4660) \rightarrow \eta_{c} \phi(1020)$, the correlation function can be written as

$$
\begin{aligned}
\Pi_{\mu \nu}(p, q)= & \frac{f_{\eta_{c}} m_{\eta_{c}}^{2}}{2 m_{c}} \frac{f_{\phi} m_{\phi} \lambda_{Y} G_{Y \eta_{c} \phi} \varepsilon_{\alpha \beta \rho \sigma} q^{\alpha} p^{\prime \rho}}{\left(p^{\prime 2}-m_{Y}^{2}\right)\left(p^{2}-m_{\eta_{c}}^{2}\right)\left(q^{2}-m_{\phi}^{2}\right)} \\
& \times\left(-g_{\mu}{ }^{\beta}+\frac{q_{\mu} q^{\beta}}{q^{2}}\right)\left(-g_{\nu}{ }^{\sigma}+\frac{p_{\nu}^{\prime} p^{\prime \sigma}}{p^{\prime 2}}\right)+\cdots \\
= & \Pi\left(p^{\prime 2}, p^{2}, q^{2}\right) \varepsilon_{\mu \nu \alpha \beta} p^{\alpha} q^{\beta}+\cdots
\end{aligned}
$$

For the decay $Y(4660) \rightarrow \chi_{c 0} \phi(1020)$, the correlation function can be written as

$$
\begin{aligned}
\Pi_{\mu \nu}(p, q)= & \frac{f_{\chi_{c 0}} m_{\chi_{c 0}} f_{\phi} m_{\phi} \lambda_{Y} G_{Y \chi_{c 0} \phi}}{\left(p^{\prime 2}-m_{Y}^{2}\right)\left(p^{2}-m_{\chi_{c 0}}^{2}\right)\left(q^{2}-m_{\phi}^{2}\right)} \\
& \times\left(-g_{\mu \alpha}+\frac{q_{\mu} q_{\alpha}}{q^{2}}\right)\left(-g_{\nu}{ }^{\alpha}+\frac{p_{\nu}^{\prime} p^{\prime \alpha}}{p^{\prime 2}}\right)+\cdots \\
= & \Pi\left(p^{\prime 2}, p^{2}, q^{2}\right) g_{\mu \nu}+\cdots
\end{aligned}
$$

For the decay $Y(4660) \rightarrow D_{S} \bar{D}_{s}$, the correlation function can be written as

$$
\begin{aligned}
\Pi_{v}(p, q)= & \frac{f_{D_{s}}^{2} m_{D_{s}}^{4}}{\left(m_{c}+m_{s}\right)^{2}} \\
& \times \frac{\lambda_{Y} G_{Y D_{s} \bar{D}_{s}}}{\left(p^{\prime 2}-m_{Y}^{2}\right)\left(p^{2}-m_{D_{s}}^{2}\right)\left(q^{2}-m_{D_{s}}^{2}\right)}(p-q)^{\alpha} \\
& \times\left(-g_{\alpha \nu}+\frac{p_{\alpha}^{\prime} p_{v}^{\prime}}{p^{\prime 2}}\right)+\cdots \\
= & \Pi\left(p^{\prime 2}, p^{2}, q^{2}\right)\left(-p_{v}\right)+\cdots .
\end{aligned}
$$

For the decay $Y(4660) \rightarrow D_{s}^{*} \bar{D}_{s}^{*}$, the correlation function can be written as

$$
\begin{aligned}
\Pi_{\alpha \beta \nu}(p, q)= & \frac{f_{D_{s}^{*}}^{2} m_{D_{s}^{*}}^{2} \lambda_{Y} G_{Y D_{s}^{*} \bar{D}_{s}^{*}}}{\left(p^{\prime 2}-m_{Y}^{2}\right)\left(p^{2}-m_{D_{s}^{*}}^{2}\right)\left(q^{2}-m_{D_{s}^{*}}^{2}\right)}(p-q)^{\sigma} \\
& \times\left(-g_{\nu \sigma}+\frac{p_{\nu}^{\prime} p_{\sigma}^{\prime}}{p^{\prime 2}}\right)\left(-g_{\alpha \rho}+\frac{p_{\alpha} p_{\rho}}{p^{2}}\right) \\
& \times\left(-g_{\beta}^{\rho}+\frac{q_{\beta} q^{\rho}}{q^{2}}\right)+\cdots \\
= & \Pi\left(p^{\prime 2}, p^{2}, q^{2}\right)\left(-g_{\alpha \beta} p_{\nu}\right)+\cdots .
\end{aligned}
$$

For the decay $Y(4660) \rightarrow D_{s} \bar{D}_{s}^{*}$, the correlation function can be written as

$$
\begin{aligned}
\Pi_{\mu \nu}(p, q)= & \frac{f_{D_{s}} m_{D_{s}}^{2}}{m_{c}+m_{s}} \frac{f_{D_{s}^{*}} m_{D_{s}^{*}} \lambda_{Y} G_{Y D_{s} \bar{D}_{s}^{*}} \varepsilon_{\alpha \beta \rho \sigma} p^{\alpha} p^{\prime \rho}}{\left(p^{\prime 2}-m_{Y}^{2}\right)\left(p^{2}-m_{D_{s}^{*}}^{2}\right)\left(q^{2}-m_{D_{s}}^{2}\right)} \\
& \times\left(-g_{\mu}{ }^{\beta}+\frac{p_{\mu} p^{\beta}}{p^{2}}\right)\left(-g_{\nu}{ }^{\sigma}+\frac{p_{\nu}^{\prime} p^{\prime \sigma}}{p^{\prime 2}}\right)+\cdots \\
= & \Pi\left(p^{\prime 2}, p^{2}, q^{2}\right)\left(-\varepsilon_{\mu \nu \alpha \beta} p^{\alpha} q^{\beta}\right)+\cdots
\end{aligned}
$$

For the decay $Y(4660) \rightarrow J / \psi \phi(1020)$, the correlation function can be written as

$$
\begin{aligned}
\Pi_{\alpha \beta \nu}(p, q)= & \frac{f_{J / \psi} m_{J / \psi} f_{\phi} m_{\phi} \lambda_{Y} G_{Y J / \psi \phi}}{\left(p^{\prime 2}-m_{Y}^{2}\right)\left(p^{2}-m_{J / \psi}^{2}\right)\left(q^{2}-m_{\phi}^{2}\right)}(p-q)^{\sigma} \\
& \times\left(-g_{\nu \sigma}+\frac{p_{\nu}^{\prime} p_{\sigma}^{\prime}}{p^{\prime 2}}\right)\left(-g_{\alpha \rho}+\frac{p_{\alpha} p_{\rho}}{p^{2}}\right) \\
& \times\left(-g_{\beta} \rho+\frac{q_{\beta} q^{\rho}}{q^{2}}\right)+\cdots \\
= & \Pi\left(p^{\prime 2}, p^{2}, q^{2}\right)\left(-g_{\alpha \beta} p_{\nu}\right)+\cdots
\end{aligned}
$$

In calculations, we observe that the hadronic coupling constant $G_{Y J / \psi \phi}$ is zero at the leading order approximation, and we will neglect the process $Y(4660) \rightarrow J / \psi \phi(1020)$.

In Eqs. (31)-(37), we have used the following definitions for the decay constants and hadronic coupling constants,

$$
\begin{aligned}
\left\langle 0\left|J_{J / \psi, \mu}(0)\right| J / \psi(p)\right\rangle & =f_{J / \psi} m_{J / \psi} \xi_{\mu}^{J / \psi}, \\
\left\langle 0\left|J_{\psi^{\prime}, \mu}(0)\right| \psi^{\prime}(p)\right\rangle & =f_{\psi^{\prime}} m_{\psi^{\prime}} \xi_{\mu}^{\psi^{\prime}}, \\
\left\langle 0\left|J_{f_{0}}(0)\right| f_{0}(p)\right\rangle & =f_{f_{0}} m_{f_{0}}, \\
\left\langle 0\left|J_{\eta_{c}}(0)\right| \eta_{c}(p)\right\rangle & =\frac{f_{\eta_{c}} m_{\eta_{c}}^{2}}{2 m_{c}}, \\
\left\langle 0\left|J_{\phi, \mu}(0)\right| \phi(p)\right\rangle & =f_{\phi} m_{\phi} \xi_{\mu}^{\phi}, \\
\left\langle 0\left|J_{\chi_{c} 0}(0)\right| \chi_{c 0}(p)\right\rangle & =f_{\chi_{c 0}} m_{\chi_{c 0}}, \\
\left\langle 0\left|J_{D_{s}}(0)\right| D_{s}(p)\right\rangle & =\frac{f_{D_{s}} m_{D_{s}}^{2}}{m_{c}+m_{s}}, \\
\left\langle 0\left|J_{D_{s}^{*}, \mu}(0)\right| D_{s}^{*}(p)\right\rangle & =f_{D_{s}^{*} m_{D_{s}^{*}} \xi_{\mu}^{D_{s}^{*}},}, \\
\left\langle 0\left|J_{\mu}(0)\right| Y(p)\right\rangle & =\lambda_{Y} \xi_{\mu}^{Y},
\end{aligned}
$$

$$
\begin{aligned}
& \left\langle J / \psi(p) f_{0}(q) \mid X\left(p^{\prime}\right)\right\rangle=i \xi_{J / \psi}^{* \alpha} \xi_{\alpha}^{Y} G_{Y J / \psi f_{0}}, \\
& \left\langle\psi^{\prime}(p) f_{0}(q) \mid X\left(p^{\prime}\right)\right\rangle=i \xi_{\psi^{\prime}}^{* \alpha} \xi_{\alpha}^{Y} G_{Y \psi^{\prime} f_{0}}, \\
& \left\langle\eta_{c}(p) \phi(q) \mid X\left(p^{\prime}\right)\right\rangle=i \varepsilon^{\alpha \beta \rho \sigma} q_{\alpha} \xi_{\beta}^{\phi *} p_{\rho}^{\prime} \xi_{\sigma}^{Y} G_{Y \eta_{c} \phi}, \\
& \left\langle\chi_{c 0}(p) \phi(q) \mid X\left(p^{\prime}\right)\right\rangle=i \xi_{\phi}^{* \alpha} \xi_{\alpha}^{Y} G_{Y \chi_{c 0} \phi}, \\
& \left\langle\bar{D}_{s}(p) D_{s}(q) \mid X\left(p^{\prime}\right)\right\rangle=i(p-q)^{\alpha} \xi_{\alpha}^{Y} G_{Y D_{s} \bar{D}_{s}}, \\
& \left\langle\bar{D}_{s}^{*}(p) D_{s}^{*}(q) \mid X\left(p^{\prime}\right)\right\rangle=i(p-q)^{\alpha} \xi_{\alpha}^{Y} \xi_{\beta} \bar{D}_{s}^{*} \xi^{D_{s}^{*} * \beta} G_{Y D_{s}^{*} \bar{D}_{s}^{*}}, \\
& \left\langle\bar{D}_{s}^{*}(p) D_{s}(q) \mid X\left(p^{\prime}\right)\right\rangle=i \varepsilon^{\alpha \beta \rho \sigma} p_{\alpha} \xi_{\beta}^{\bar{D}_{s}^{*} *} p_{\rho}^{\prime} \xi_{\sigma}^{Y} G_{Y D_{s} \bar{D}_{s}^{*}}, \\
& \left\langle J / \psi(p) \phi(q) \mid X\left(p^{\prime}\right)\right\rangle=i(p-q)^{\alpha} \xi_{\alpha}^{Y} \xi_{\beta}^{J / \psi^{*}} \xi^{\phi * \beta} G_{Y J / \psi \phi},
\end{aligned}
$$

where the $\xi_{\mu}^{J / \psi}, \xi_{\mu}^{\psi^{\prime}}, \xi_{\mu}^{\phi}, \xi_{\mu}^{D_{s}^{*}}, \xi_{\mu}^{Y}$ are the polarization vectors, the $G_{Y J / \psi f_{0}}, G_{Y \psi^{\prime} f_{0}}, G_{Y \eta_{c} \phi}, G_{Y \chi_{c 0} \phi}, G_{Y D_{s} \bar{D}_{s}}, G_{Y D_{s}^{*} \bar{D}_{s}^{*}}$, $G_{Y D_{s} \bar{D}_{s}^{*}}, G_{Y J / \psi \phi}$ are the hadronic coupling constants.

We study the components $\Pi\left(p^{\prime 2}, p^{2}, q^{2}\right)$ of the correlation functions, and carry out the operator product expansion up to the vacuum condensates of dimension 5 and neglect the tiny contributions of the gluon condensate. Then we obtain 
Eur. Phys. J. C (2019) 79:184

Page 7 of $13 \quad \mathbf{1 8 4}$

the QCD spectral densities through dispersion relation and use Eqs. (17) and (18) to obtain the QCD sum rules for the hadronic coupling constants,

$$
\begin{aligned}
& \frac{f_{J / \psi} m_{J / \psi} f_{f_{0}} m_{f_{0}} \lambda_{Y} G_{Y J / \psi f_{0}}}{m_{Y}^{2}-m_{J / \psi}^{2}}\left[\exp \left(-\frac{m_{J / \psi}^{2}}{T_{1}^{2}}\right)\right. \\
& \left.-\exp \left(-\frac{m_{Y}^{2}}{T_{1}^{2}}\right)\right] \exp \left(-\frac{m_{f_{0}}^{2}}{T_{2}^{2}}\right) \\
& +\left(C_{Y^{\prime} J / \psi}+C_{Y^{\prime} f_{0}}\right) \exp \left(-\frac{m_{J / \psi}^{2}}{T_{1}^{2}}-\frac{m_{f_{0}}^{2}}{T_{2}^{2}}\right) \\
& =-\frac{1}{32 \sqrt{2} \pi^{4}} \int_{4 m_{c}^{2}}^{s_{J / \psi}^{0}} d s \int_{0}^{s_{f_{0}}^{0}} d u u s \sqrt{1-\frac{4 m_{c}^{2}}{s}}\left(1+\frac{2 m_{c}^{2}}{s}\right) \\
& \times \exp \left(-\frac{s}{T_{1}^{2}}-\frac{u}{T_{2}^{2}}\right) \\
& -\frac{m_{s}\langle\bar{s} s\rangle}{4 \sqrt{2} \pi^{2}} \int_{4 m_{c}^{2}}^{s_{J / \psi}^{0}} d s s \sqrt{1-\frac{4 m_{c}^{2}}{s}}\left(1+\frac{2 m_{c}^{2}}{s}\right) \exp \left(-\frac{s}{T_{1}^{2}}\right) \\
& -\frac{m_{s}\left\langle\bar{s} g_{s} \sigma G s\right\rangle}{12 \sqrt{2} \pi^{2} T_{2}^{2}} \int_{4 m_{c}^{2}}^{s_{J / \psi}^{0}} d s \sqrt{1-\frac{4 m_{c}^{2}}{s}}\left(s+2 m_{c}^{2}\right) \exp \left(-\frac{s}{T_{1}^{2}}\right) \\
& +\frac{m_{s}\left\langle\bar{s} g_{s} \sigma G s\right\rangle}{48 \sqrt{2} \pi^{2}} \int_{4 m_{c}^{2}}^{s_{J / \psi}^{0}} d s \frac{s-12 m_{c}^{2}}{\sqrt{s\left(s-4 m_{c}^{2}\right)}} \exp \left(-\frac{s}{T_{1}^{2}}\right),
\end{aligned}
$$

$$
\begin{aligned}
\frac{f_{\eta_{c}} m_{\eta_{c}}^{2}}{2 m_{c}} \frac{f_{\phi} m_{\phi} \lambda_{Y} G_{Y \eta_{c} \phi}}{m_{Y}^{2}-m_{\eta_{c}}^{2}}\left[\exp \left(-\frac{m_{\eta_{c}}^{2}}{T_{1}^{2}}\right)\right. \\
\left.\quad-\exp \left(-\frac{m_{Y}^{2}}{T_{1}^{2}}\right)\right] \exp \left(-\frac{m_{\phi}^{2}}{T_{2}^{2}}\right) \\
+\left(C_{Y^{\prime} \eta_{c}}+C_{Y^{\prime} \phi}\right) \exp \left(-\frac{m_{\eta_{c}}^{2}}{T_{1}^{2}}-\frac{m_{\phi}^{2}}{T_{2}^{2}}\right) \\
=\frac{3 m_{s} m_{c}}{16 \sqrt{2} \pi^{4}} \int_{4 m_{c}^{2}}^{s_{\eta_{c}}^{0}} d s \int_{0}^{s_{\phi}^{0}} d u \sqrt{1-\frac{4 m_{c}^{2}}{s}} \exp \left(-\frac{s}{T_{1}^{2}}-\frac{u}{T_{2}^{2}}\right) \\
-\frac{m_{c}\langle\bar{s} s\rangle}{2 \sqrt{2} \pi^{2}} \int_{4 m_{c}^{2}}^{s_{\eta_{c}}^{0}} d s \sqrt{1-\frac{4 m_{c}^{2}}{s} \exp \left(-\frac{s}{T_{1}^{2}}\right)} \\
+\frac{m_{c}\left\langle\bar{s} g_{s} \sigma G s\right\rangle}{6 \sqrt{2} \pi^{2} T_{2}^{2}} \int_{4 m_{c}^{2}}^{s_{\eta_{c}}^{0}} d s \sqrt{1-\frac{4 m_{c}^{2}}{s}} \exp \left(-\frac{s}{T_{1}^{2}}\right) \\
\quad-\frac{m_{c}\left\langle\bar{s} g_{s} \sigma G s\right\rangle}{24 \sqrt{2} \pi^{2}} \int_{4 m_{c}^{2}}^{s_{\eta_{c}}^{0}} d s \frac{1}{\sqrt{s\left(s-4 m_{c}^{2}\right)}} \exp \left(-\frac{s}{T_{1}^{2}}\right)
\end{aligned}
$$

(41)

$$
\begin{aligned}
& \frac{f_{\chi_{c 0}} m_{\chi_{c 0}} f_{\phi} m_{\phi} \lambda_{Y} G_{Y \chi_{c 0} \phi}}{m_{Y}^{2}-m_{\chi_{c 0}}^{2}}\left[\exp \left(-\frac{m_{\chi_{c 0}}^{2}}{T_{1}^{2}}\right)\right. \\
& \left.-\exp \left(-\frac{m_{Y}^{2}}{T_{1}^{2}}\right)\right] \exp \left(-\frac{m_{\phi}^{2}}{T_{2}^{2}}\right) \\
& +\left(C_{Y^{\prime} \chi_{c 0}}+C_{Y^{\prime} \phi}\right) \exp \left(-\frac{m_{\chi_{c 0}}^{2}}{T_{1}^{2}}-\frac{m_{\phi}^{2}}{T_{2}^{2}}\right) \\
& =\frac{1}{32 \sqrt{2} \pi^{4}} \int_{4 m_{c}^{2}}^{s_{\chi_{c 0}}^{0}} d s \int_{0}^{s_{\phi}^{0}} d u u s \sqrt{1-\frac{4 m_{c}^{2}}{s}} \\
& \times\left(1-\frac{4 m_{c}^{2}}{s}\right) \exp \left(-\frac{s}{T_{1}^{2}}-\frac{u}{T_{2}^{2}}\right) \\
& -\frac{m_{s}\langle\bar{s} s\rangle}{4 \sqrt{2} \pi^{2}} \int_{4 m_{c}^{2}}^{s_{\chi_{c 0}}^{0}} d s s \sqrt{1-\frac{4 m_{c}^{2}}{s}} \\
& \times\left(1-\frac{4 m_{c}^{2}}{s}\right) \exp \left(-\frac{s}{T_{1}^{2}}\right) \\
& +\frac{m_{s}\left\langle\bar{s} g_{s} \sigma G s\right\rangle}{48 \sqrt{2} \pi^{2} T_{2}^{2}} \int_{4 m_{c}^{2}}^{s_{\chi_{c 0}}^{0}} d s s \sqrt{1-\frac{4 m_{c}^{2}}{s}}\left(1-\frac{4 m_{c}^{2}}{s}\right) \exp \left(-\frac{s}{T_{1}^{2}}\right) \\
& -\frac{m_{s}\left\langle\bar{s} g_{s} \sigma G s\right\rangle}{24 \sqrt{2} \pi^{2}} \int_{4 m_{c}^{2}}^{s_{\chi_{c 0}}^{0}} d s \frac{s-6 m_{c}^{2}}{\sqrt{s\left(s-4 m_{c}^{2}\right)}} \exp \left(-\frac{s}{T_{1}^{2}}\right) \text {, } \\
& \frac{f_{D_{s}}^{2} m_{D_{s}}^{4}}{\left(m_{c}+m_{s}\right)^{2}} \frac{\lambda_{Y} G_{Y D_{s} \bar{D}_{s}}}{4\left(\tilde{m}_{Y}^{2}-m_{D_{s}}^{2}\right)}\left[\exp \left(-\frac{m_{D_{s}}^{2}}{T_{1}^{2}}\right)\right. \\
& \left.-\exp \left(-\frac{\tilde{m}_{Y}^{2}}{T_{1}^{2}}\right)\right] \exp \left(-\frac{m_{D_{s}}^{2}}{T_{2}^{2}}\right) \\
& +\left(C_{Y^{\prime} \bar{D}_{s}}+C_{Y^{\prime} D_{s}}\right) \exp \left(-\frac{m_{D_{s}}^{2}}{T_{1}^{2}}-\frac{m_{D_{s}}^{2}}{T_{2}^{2}}\right) \\
& =\frac{3 m_{c}}{64 \sqrt{2} \pi^{4}} \int_{m_{c}^{2}}^{s_{D_{s}}^{0}} d s \int_{m_{c}^{2}}^{s_{D_{s}}^{0}} d u u\left(1-\frac{m_{c}^{2}}{s}\right)^{2} \\
& \times\left(1-\frac{m_{c}^{2}}{u}\right)^{2} \exp \left(-\frac{s}{T_{1}^{2}}-\frac{u}{T_{2}^{2}}\right) \\
& +\frac{3 m_{s}}{64 \sqrt{2} \pi^{4}} \int_{m_{c}^{2}}^{s_{D_{s}}^{0}} d s \int_{m_{c}^{2}}^{s_{D_{s}}^{0}} d u u\left(1-\frac{m_{c}^{2}}{s}\right) \\
& \times\left(1-\frac{m_{c}^{2}}{u}\right)\left(1+\frac{m_{c}^{2}}{s}+\frac{m_{c}^{2}}{u}-\frac{3 m_{c}^{4}}{u s}\right) \exp \left(-\frac{s}{T_{1}^{2}}-\frac{u}{T_{2}^{2}}\right) \\
& -\frac{\langle\bar{s} s\rangle}{8 \sqrt{2} \pi^{2}} \int_{m_{c}^{2}}^{s_{D_{s}}^{0}} d u\left[u\left(1-\frac{m_{c}^{2}}{u}\right)^{2}\right. \\
& \left.+2 m_{s} m_{c}\left(1-\frac{m_{c}^{2}}{u}\right)\right] \exp \left(-\frac{m_{c}^{2}}{T_{1}^{2}}-\frac{u}{T_{2}^{2}}\right) \\
& -\frac{\langle\bar{s} s\rangle}{8 \sqrt{2} \pi^{2}} \int_{m_{c}^{2}}^{s_{D_{s}}^{0}} d s\left[m_{c}^{2}\left(1-\frac{m_{c}^{2}}{s}\right)^{2}\right. \\
& \left.+m_{s} m_{c}\left(1-\frac{m_{c}^{4}}{s^{2}}\right)\right] \exp \left(-\frac{s}{T_{1}^{2}}-\frac{m_{c}^{2}}{T_{2}^{2}}\right)
\end{aligned}
$$

Springer 


$$
\begin{aligned}
& +\frac{m_{s} m_{c}\langle\bar{s} s\rangle}{16 \sqrt{2} \pi^{2} T_{1}^{2}} \int_{m_{c}^{2}}^{s_{D_{s}}^{0}} d u u\left(1-\frac{m_{c}^{2}}{u}\right)^{2} \exp \left(-\frac{m_{c}^{2}}{T_{1}^{2}}-\frac{u}{T_{2}^{2}}\right) \\
& +\frac{m_{s} m_{c}\langle\bar{s} s\rangle}{16 \sqrt{2} \pi^{2}} \int_{m_{c}^{2}}^{s_{D_{s}}^{0}} d s\left(1-\frac{m_{c}^{2}}{s}\right)^{2} \\
& \times\left(1+\frac{m_{c}^{2}}{T_{2}^{2}}\right) \exp \left(-\frac{s}{T_{1}^{2}}-\frac{m_{c}^{2}}{T_{2}^{2}}\right) \\
& +\frac{m_{c}^{2}\left\langle\bar{s} g_{s} \sigma G s\right\rangle}{32 \sqrt{2} \pi^{2} T_{1}^{4}} \int_{m_{c}^{2}}^{s_{D_{s}}^{0}} d u\left[u\left(1-\frac{m_{c}^{2}}{u}\right)^{2}\right. \\
& \left.+2 m_{s} m_{c}\left(1-\frac{m_{c}^{2}}{u}\right)\right] \exp \left(-\frac{m_{c}^{2}}{T_{1}^{2}}-\frac{u}{T_{2}^{2}}\right) \\
& -\frac{\left\langle\bar{s} g_{s} \sigma G s\right\rangle}{16 \sqrt{2} \pi^{2} T_{2}^{2}} \int_{m_{c}^{2}}^{s_{D_{s}}^{0}} d s\left[m_{c}^{2}\left(1-\frac{m_{c}^{2}}{s}\right)^{2}\right. \\
& \left.+m_{s} m_{c}\left(1-\frac{m_{c}^{4}}{s^{2}}\right)\right]\left(1-\frac{m_{c}^{2}}{2 T_{2}^{2}}\right) \exp \left(-\frac{s}{T_{1}^{2}}-\frac{m_{c}^{2}}{T_{2}^{2}}\right) \\
& -\frac{m_{s} m_{c}^{3}\left\langle\bar{s} g_{s} \sigma G s\right\rangle}{96 \sqrt{2} \pi^{2} T_{1}^{6}} \int_{m_{c}^{2}}^{s_{D_{s}}^{0}} d u u\left(1-\frac{m_{c}^{2}}{u}\right)^{2} \exp \left(-\frac{m_{c}^{2}}{T_{1}^{2}}-\frac{u}{T_{2}^{2}}\right) \\
& -\frac{m_{s} m_{c}^{5}\left\langle\bar{s} g_{s} \sigma G s\right\rangle}{96 \sqrt{2} \pi^{2} T_{2}^{6}} \int_{m_{c}^{2}}^{s_{D_{s}}^{0}} d s\left(1-\frac{m_{c}^{2}}{s}\right)^{2} \exp \left(-\frac{s}{T_{1}^{2}}-\frac{m_{c}^{2}}{T_{2}^{2}}\right) \\
& -\frac{\left\langle\bar{s} g_{s} \sigma G s\right\rangle}{192 \sqrt{2} \pi^{2}} \int_{m_{c}^{2}}^{s_{D_{s}}^{0}} d u\left[\left(1-\frac{m_{c}^{2}}{u}\right)\left(3-\frac{m_{c}^{2}}{u}\right)\right. \\
& \left.+\frac{6 m_{s} m_{c}}{u}\right] \exp \left(-\frac{m_{c}^{2}}{T_{1}^{2}}-\frac{u}{T_{2}^{2}}\right) \\
& -\frac{\left\langle\bar{s} g_{s} \sigma G s\right\rangle}{96 \sqrt{2} \pi^{2}} \int_{m_{c}^{2}}^{s_{D_{s}}^{0}} d s\left[\frac{m_{c}^{2}}{s}\left(1-\frac{m_{c}^{2}}{s}\right)\left(6-\frac{m_{c}^{2}}{s}\right)\right. \\
& \left.-\frac{3 m_{s} m_{c}^{3}}{s^{2}}\right] \exp \left(-\frac{s}{T_{1}^{2}}-\frac{m_{c}^{2}}{T_{2}^{2}}\right) \\
& -\frac{\left\langle\bar{s} g_{s} \sigma G s\right\rangle}{192 \sqrt{2} \pi^{2}} \int_{m_{c}^{2}}^{s_{D_{s}}^{0}} d u\left[\left(3-\frac{m_{c}^{4}}{u^{2}}\right)\right. \\
& \left.+\frac{6 m_{s} m_{c}}{u} \frac{u+m_{c}^{2}}{u-m_{c}^{2}}\right] \exp \left(-\frac{m_{c}^{2}}{T_{1}^{2}}-\frac{u}{T_{2}^{2}}\right) \\
& -\frac{\left\langle\bar{s} g_{s} \sigma G s\right\rangle}{96 \sqrt{2} \pi^{2}} \int_{m_{c}^{2}}^{s_{D_{s}}^{0}} d s\left(\frac{m_{c}^{6}}{s^{3}}\right. \\
& \left.+\frac{3 m_{s} m_{c}}{s^{2}} \frac{s^{2}+m_{c}^{4}}{s-m_{c}^{2}}\right) \exp \left(-\frac{s}{T_{1}^{2}}-\frac{m_{c}^{2}}{T_{2}^{2}}\right),
\end{aligned}
$$$$
+\left(C_{Y^{\prime} \bar{D}_{s}^{*}}+C_{Y^{\prime} D_{s}^{*}}\right) \exp \left(-\frac{m_{D_{s}^{*}}^{2}}{T_{1}^{2}}-\frac{m_{D_{s}^{*}}^{2}}{T_{2}^{2}}\right)
$$$$
=\frac{m_{c}}{64 \sqrt{2} \pi^{4}} \int_{m_{c}^{2}}^{s_{D_{s}^{*}}^{0}} d s \int_{m_{c}^{2}}^{s_{D_{s}^{*}}^{0}} d u\left(2 u+m_{c}^{2}\right)\left(1-\frac{m_{c}^{2}}{s}\right)^{2}
$$$$
\times\left(1-\frac{m_{c}^{2}}{u}\right)^{2} \exp \left(-\frac{s}{T_{1}^{2}}-\frac{u}{T_{2}^{2}}\right)
$$$$
+\frac{m_{s}}{64 \sqrt{2} \pi^{4}} \int_{m_{c}^{2}}^{s_{D_{s}^{*}}^{0}} d s \int_{m_{c}^{2}}^{s_{D_{s}^{*}}^{0}} d u u\left(1-\frac{m_{c}^{2}}{s}\right)\left(1-\frac{m_{c}^{2}}{u}\right)
$$$$
\times\left(2+\frac{2 m_{c}^{2}}{s}+\frac{5 m_{c}^{2}}{u}-\frac{m_{c}^{4}}{u^{2}}\right.
$$$$
\left.-\frac{7 m_{c}^{4}}{u s}-\frac{m_{c}^{6}}{u s^{2}}\right) \exp \left(-\frac{s}{T_{1}^{2}}-\frac{u}{T_{2}^{2}}\right)
$$$$
-\frac{\langle\bar{s} s\rangle}{24 \sqrt{2} \pi^{2}} \int_{m_{c}^{2}}^{s_{D_{s}^{*}}^{0}} d u\left[\left(2 u+m_{c}^{2}\right)\left(1-\frac{m_{c}^{2}}{u}\right)^{2}\right.
$$$$
\left.+6 m_{s} m_{c}\left(1-\frac{m_{c}^{2}}{u}\right)\right] \exp \left(-\frac{m_{c}^{2}}{T_{1}^{2}}-\frac{u}{T_{2}^{2}}\right)
$$$$
-\frac{\langle\bar{s} s\rangle}{8 \sqrt{2} \pi^{2}} \int_{m_{c}^{2}}^{s_{D_{s}^{*}}^{0}} d s\left[m_{c}^{2}\left(1-\frac{m_{c}^{2}}{s}\right)^{2}\right.
$$$$
\left.+m_{s} m_{c}\left(1-\frac{m_{c}^{4}}{s^{2}}\right)\right] \exp \left(-\frac{s}{T_{1}^{2}}-\frac{m_{c}^{2}}{T_{2}^{2}}\right)
$$$$
+\frac{m_{s} m_{c}\langle\bar{s} s\rangle}{48 \sqrt{2} \pi^{2} T_{1}^{2}} \int_{m_{c}^{2}}^{s_{D_{s}^{*}}^{0}} d u\left(2 u+m_{c}^{2}\right)
$$$$
\times\left(1-\frac{m_{c}^{2}}{u}\right)^{2} \exp \left(-\frac{m_{c}^{2}}{T_{1}^{2}}-\frac{u}{T_{2}^{2}}\right)
$$$$
+\frac{m_{s} m_{c}^{3}\langle\bar{s} s\rangle}{16 \sqrt{2} \pi^{2} T_{2}^{2}} \int_{m_{c}^{2}}^{s_{D_{s}^{*}}^{0}} d s\left(1-\frac{m_{c}^{2}}{s}\right)^{2} \exp \left(-\frac{s}{T_{1}^{2}}-\frac{m_{c}^{2}}{T_{2}^{2}}\right)
$$$$
+\frac{\left\langle\bar{s} g_{s} \sigma G s\right\rangle}{288 \sqrt{2} \pi^{2} T_{1}^{2}} \int_{m_{c}^{2}}^{s_{D_{s}^{*}}^{0}} d u\left[\left(2 u+m_{c}^{2}\right)\left(1-\frac{m_{c}^{2}}{u}\right)^{2}\right.
$$$$
\left.+6 m_{s} m_{c}\left(1-\frac{m_{c}^{2}}{u}\right)\right]\left(4+\frac{3 m_{c}^{2}}{T_{1}^{2}}\right) \exp \left(-\frac{m_{c}^{2}}{T_{1}^{2}}-\frac{u}{T_{2}^{2}}\right)
$$$$
+\frac{m_{c}^{2}\left\langle\bar{s} g_{s} \sigma G s\right\rangle}{32 \sqrt{2} \pi^{2} T_{2}^{4}} \int_{m_{c}^{2}}^{s_{D_{s}^{*}}^{0}} d s\left[m_{c}^{2}\left(1-\frac{m_{c}^{2}}{s}\right)^{2}\right.
$$$$
\left.+m_{s} m_{c}\left(1-\frac{m_{c}^{4}}{s^{2}}\right)\right] \exp \left(-\frac{s}{T_{1}^{2}}-\frac{m_{c}^{2}}{T_{2}^{2}}\right)
$$$$
-\frac{m_{s} m_{c}^{3}\left\langle\bar{s} g_{s} \sigma G s\right\rangle}{288 \sqrt{2} \pi^{2} T_{1}^{6}} \int_{m_{c}^{2}}^{s_{D_{s}^{*}}^{0}} d u\left(2 u+m_{c}^{2}\right)
$$$$
\begin{gathered}
\frac{f_{D_{s}^{*}}^{2} m_{D_{s}^{*}}^{2} \lambda_{Y} G_{Y D_{s}^{*} \bar{D}_{s}^{*}}}{4\left(\tilde{m}_{Y}^{2}-m_{D_{s}^{*}}^{2}\right)}\left[\exp \left(-\frac{m_{D_{s}^{*}}^{2}}{T_{1}^{2}}\right)\right. \\
\left.-\exp \left(-\frac{\widetilde{m}_{Y}^{2}}{T_{1}^{2}}\right)\right] \exp \left(-\frac{m_{D_{s}^{*}}^{2}}{T_{2}^{2}}\right)
\end{gathered}
$$

$\times\left(1-\frac{m_{c}^{2}}{u}\right)^{2} \exp \left(-\frac{m_{c}^{2}}{T_{1}^{2}}-\frac{u}{T_{2}^{2}}\right)$ 


$$
\begin{aligned}
& +\frac{m_{s} m_{c}\left\langle\bar{s} g_{s} \sigma G s\right\rangle}{96 \sqrt{2} \pi^{2} T_{2}^{2}} \int_{m_{c}^{2}}^{s_{D_{s}^{*}}^{0}} d s \\
& \times\left(1-\frac{m_{c}^{2}}{s}\right)^{2}\left(1+\frac{m_{c}^{2}}{T_{2}^{2}}-\frac{m_{c}^{4}}{T_{2}^{4}}\right) \exp \left(-\frac{s}{T_{1}^{2}}-\frac{m_{c}^{2}}{T_{2}^{2}}\right) \\
& -\frac{\left\langle\bar{s} g_{s} \sigma G s\right\rangle}{96 \sqrt{2} \pi^{2}} \int_{m_{c}^{2}}^{s_{D_{s}^{*}}^{0}} d u\left[\left(1-\frac{m_{c}^{2}}{u}\right)\right. \\
& \left.+\frac{3 m_{s} m_{c}}{u}\right] \exp \left(-\frac{m_{c}^{2}}{T_{1}^{2}}-\frac{u}{T_{2}^{2}}\right) \\
& -\frac{\left\langle\bar{s} g_{s} \sigma G s\right\rangle}{96 \sqrt{2} \pi^{2}} \int_{m_{c}^{2}}^{s_{D_{s}^{*}}^{0}} d s\left[\frac{m_{c}^{2}}{s}\left(1-\frac{m_{c}^{2}}{s}\right)\left(6-\frac{m_{c}^{2}}{s}\right)\right. \\
& \left.-\frac{\left.3 m_{s} m_{c}^{3}\right]}{s^{2}}\right] \exp \left(-\frac{s}{T_{1}^{2}}-\frac{m_{c}^{2}}{T_{2}^{2}}\right) \\
& +\frac{\left\langle\bar{s} g_{s} \sigma G s\right\rangle}{96 \sqrt{2} \pi^{2}} \int_{m_{c}^{2}}^{s_{D_{s}^{*}}^{0}} d u\left(1+\frac{m_{s} m_{c}}{u} \frac{u+m_{c}^{2}}{u-m_{c}^{2}}\right) \\
& \left.+\frac{m_{s} m_{c}}{s^{2}} \frac{s^{2}+m_{c}^{4}}{s-m_{s}^{2}}\right) \exp \left(-\frac{s}{T_{1}^{2}}-\frac{m_{c}^{2}}{T_{2}^{2}}\right) \\
& m_{c}^{2} \\
& T_{1}^{2}
\end{aligned}
$$

$\frac{f_{D_{s}} m_{D_{s}}^{2}}{m_{c}+m_{s}} \frac{f_{D_{s}^{*}} m_{D_{s}^{*}} \lambda_{Y} G_{Y D_{s} \bar{D}_{s}^{*}}}{4\left(\tilde{m}_{Y}^{2}-m_{D_{s}^{*}}^{2}\right)}\left[\exp \left(-\frac{m_{D_{s}^{*}}^{2}}{T_{1}^{2}}\right)\right.$$$
\left.-\exp \left(-\frac{\tilde{m}_{Y}^{2}}{T_{1}^{2}}\right)\right] \exp \left(-\frac{m_{D_{s}}^{2}}{T_{2}^{2}}\right)
$$$$
+\left(C_{Y^{\prime} \bar{D}_{s}^{*}}+C_{Y^{\prime} D_{s}}\right) \exp \left(-\frac{m_{D_{s}^{*}}^{2}}{T_{1}^{2}}-\frac{m_{D_{s}}^{2}}{T_{2}^{2}}\right)
$$$$
=-\frac{\left\langle\bar{s} g_{s} \sigma G s\right\rangle}{32 \sqrt{2} \pi^{2}} \int_{m_{c}^{2}}^{s_{D_{s}}^{0}} d u\left[\frac{m_{c}}{u}\left(1-\frac{m_{c}^{2}}{u}\right)\right.
$$$$
\left.+\frac{2 m_{s} m_{c}^{2}}{3 u^{2}}\right] \exp \left(-\frac{m_{c}^{2}}{T_{1}^{2}}-\frac{u}{T_{2}^{2}}\right)
$$$$
+\frac{\left\langle\bar{s} g_{s} \sigma G s\right\rangle}{32 \sqrt{2} \pi^{2}} \int_{m_{c}^{2}}^{s^{0}} d s\left[\frac{m_{c}}{s}\left(1-\frac{m_{c}^{2}}{s}\right)\right.
$$$$
\left.-\frac{2 m_{s} m_{c}^{2}}{3 s^{2}}\right] \exp \left(-\frac{s}{T_{1}^{2}}-\frac{m_{c}^{2}}{T_{2}^{2}}\right)
$$$$
-\frac{\left\langle\bar{s} g_{s} \sigma G s\right\rangle}{48 \sqrt{2} \pi^{2}} \int_{m_{c}^{2}}^{s_{D_{s}}^{0}} d u\left(\frac{m_{c}^{3}}{u^{2}}+\frac{m_{s}}{2 u^{2}} \frac{u^{2}+m_{c}^{4}}{u-m_{c}^{2}}\right)
$$$$
\times \exp \left(-\frac{m_{c}^{2}}{T_{1}^{2}}-\frac{u}{T_{2}^{2}}\right)-\frac{\left\langle\bar{s} g_{s} \sigma G s\right\rangle}{96 \sqrt{2} \pi^{2}}
$$$$
\times \int_{m_{c}^{2}}^{s_{D_{s}^{*}}^{0}} d s\left(\frac{m_{s}}{s^{2}} \frac{s^{2}+m_{c}^{4}}{s-m_{c}^{2}}\right) \exp \left(-\frac{s}{T_{1}^{2}}-\frac{m_{c}^{2}}{T_{2}^{2}}\right),
$$

$$
\begin{aligned}
& \frac{f_{J / \psi} m_{J / \psi} f_{f_{0}} m_{f_{0}} \lambda_{Y} G_{Y J / \psi f_{0}}}{m_{Y}^{2}-m_{J / \psi}^{2}}\left[\exp \left(-\frac{m_{J / \psi}^{2}}{T_{1}^{2}}\right)\right. \\
& \left.-\exp \left(-\frac{m_{Y}^{2}}{T_{1}^{2}}\right)\right] \exp \left(-\frac{m_{f_{0}}^{2}}{T_{2}^{2}}\right) \\
& +\left(C_{Y^{\prime} J / \psi}+C_{Y^{\prime} f_{0}}\right) \exp \left(-\frac{m_{J / \psi}^{2}}{T_{1}^{2}}-\frac{m_{f_{0}}^{2}}{T_{2}^{2}}\right) \\
& +\frac{f_{\psi^{\prime}} m_{\psi^{\prime}} f_{f_{0}} m_{f_{0}} \lambda_{Y} G_{Y \psi^{\prime} f_{0}}}{m_{Y}^{2}-m_{\psi^{\prime}}^{2}}\left[\exp \left(-\frac{m_{\psi^{\prime}}^{2}}{T_{1}^{2}}\right)\right. \\
& \left.-\exp \left(-\frac{m_{Y}^{2}}{T_{1}^{2}}\right)\right] \exp \left(-\frac{m_{f_{0}}^{2}}{T_{2}^{2}}\right) \\
& +\left(C_{Y^{\prime} \psi^{\prime}}+\widetilde{C}_{Y^{\prime} f_{0}}\right) \exp \left(-\frac{m_{\psi^{\prime}}^{2}}{T_{1}^{2}}-\frac{m_{f_{0}}^{2}}{T_{2}^{2}}\right) \\
& =-\frac{1}{32 \sqrt{2} \pi^{4}} \int_{4 m_{c}^{2}}^{s_{\psi^{\prime}}^{0}} d s \int_{0}^{s_{f_{0}}^{0}} d u u s \sqrt{1-\frac{4 m_{c}^{2}}{s}} \\
& \times\left(1+\frac{2 m_{c}^{2}}{s}\right) \exp \left(-\frac{s}{T_{1}^{2}}-\frac{u}{T_{2}^{2}}\right) \\
& -\frac{m_{s}\langle\bar{s} s\rangle}{4 \sqrt{2} \pi^{2}} \int_{4 m_{c}^{2}}^{s_{\psi^{\prime}}^{0}} d s s \sqrt{1-\frac{4 m_{c}^{2}}{s}}\left(1+\frac{2 m_{c}^{2}}{s}\right) \exp \left(-\frac{s}{T_{1}^{2}}\right) \\
& -\frac{m_{s}\left\langle\bar{s} g_{s} \sigma G s\right\rangle}{12 \sqrt{2} \pi^{2} T_{2}^{2}} \int_{4 m_{c}^{2}}^{s_{\psi^{\prime}}^{0}} d s \sqrt{1-\frac{4 m_{c}^{2}}{s}}\left(s+2 m_{c}^{2}\right) \exp \left(-\frac{s}{T_{1}^{2}}\right) \\
& +\frac{m_{s}\left\langle\bar{s} g_{s} \sigma G s\right\rangle}{48 \sqrt{2} \pi^{2}} \int_{4 m_{c}^{2}}^{s_{\psi^{\prime}}^{0}} d s \frac{s-12 m_{c}^{2}}{\sqrt{s\left(s-4 m_{c}^{2}\right)}} \exp \left(-\frac{s}{T_{1}^{2}}\right) \text {, }
\end{aligned}
$$

where $\tilde{m}_{Y}^{2}=\frac{m_{Y}^{2}}{4}$. In calculations, we observe that there appears divergence due to the endpoint $s=4 m_{c}^{2}, s=m_{c}^{2}$ and $u=m_{c}^{2}$, we can avoid the endpoint divergence with the simple replacement $\frac{1}{s-4 m_{c}^{2}} \rightarrow \frac{1}{s-4 m_{c}^{2}+4 m_{s}^{2}}, \frac{1}{u-m_{c}^{2}} \rightarrow \frac{1}{u-m_{c}^{2}+4 m_{s}^{2}}$ and $\frac{1}{s-m_{c}^{2}} \rightarrow \frac{1}{s-m_{c}^{2}+4 m_{s}^{2}}$ by adding a small squared $s$-quark mass $4 m_{s}^{2}$.

The hadronic parameters are taken as $m_{J / \psi}=3.0969$ $\mathrm{GeV}, m_{\phi}=1.019461 \mathrm{GeV}, m_{\eta_{c}}=2.9839 \mathrm{GeV}, m_{f_{0}}=$ $0.990 \mathrm{GeV}, m_{D_{s}}=1.969 \mathrm{GeV}, m_{D_{s}^{*}}=2.1122 \mathrm{GeV}$, $m_{\chi_{c 0}}=3.41471 \mathrm{GeV}, m_{\psi^{\prime}}=3.686097 \mathrm{GeV}, m_{\pi^{+}}=$ $0.13957 \mathrm{GeV}, f_{\psi^{\prime}}=0.295 \mathrm{GeV}, \sqrt{s_{J / \psi}^{0}}=3.6 \mathrm{GeV}$, $\sqrt{s_{\eta_{c}}^{0}}=3.5 \mathrm{GeV}, \sqrt{s_{\psi^{\prime}}^{0}}=4.0 \mathrm{GeV}, \sqrt{s_{D_{s}}^{0}}=2.5 \mathrm{GeV}$, $\sqrt{s_{D_{s}^{*}}^{0}}=2.6 \mathrm{GeV}, \sqrt{s_{\chi_{c 0}}^{0}}=3.9 \mathrm{GeV}[11], f_{J / \psi}=$ $0.418 \mathrm{GeV}, f_{\eta_{c}}=0.387 \mathrm{GeV}[42], f_{\phi}=0.253 \mathrm{GeV}$, $\sqrt{s_{\phi}^{0}}=1.5 \mathrm{GeV}$ [43], $f_{f_{0}}=0.180 \mathrm{GeV}, \sqrt{s_{f_{0}}^{0}}=1.3 \mathrm{GeV}$ [44], $f_{D_{s}}=0.240 \mathrm{GeV}, f_{D_{s}^{*}}=0.308 \mathrm{GeV}[45,46]$, $f_{\chi_{c 0}}=0.359 \mathrm{GeV}$ [47], $m_{Y}=4.652 \mathrm{GeV}$ [3], $\lambda_{Y}=$ $6.72 \times 10^{-2} \mathrm{GeV}^{5}$ [24]. In Ref. [24], we obtain the values 
Table 3 The Borel windows, hadronic coupling constants, partial decay widths of the $Y(4660)$

\begin{tabular}{llll}
\hline & $T^{2}\left(\mathrm{GeV}^{2}\right)$ & $\left|G_{A B C}\right|$ & $\Gamma(\mathrm{MeV})$ \\
\hline$Y(4660) \rightarrow J / \psi f_{0}(980)$ & $3.2-4.2$ & $1.37_{-1.06}^{+1.16} \mathrm{GeV}$ & $3.5_{-3.4}^{+8.5}$ \\
$Y(4660) \rightarrow \eta_{c} \phi(1020)$ & $4.3-5.3$ & $0.98_{-0.25}^{+0.27} \mathrm{GeV}^{-1}$ & $31.6_{-14.0}^{+19.8}$ \\
$Y(4660) \rightarrow \chi_{c 0} \phi(1020)$ & $3.6-4.6$ & $1.17_{-0.95}^{+1.07} \mathrm{GeV}$ & $1.7_{-1.6}^{+4.5}$ \\
$Y(4660) \rightarrow D_{s} \bar{D}_{s}$ & $1.9-2.7$ & $1.36_{-0.33}^{+0.39}$ & $8.6_{-3.7}^{+5.6}$ \\
$Y(4660) \rightarrow D_{s}^{*} \bar{D}_{s}^{*}$ & $2.5-3.3$ & $1.57_{-0.50}^{+0.53}$ & $22.5_{-11.7}^{+17.8}$ \\
$Y(4660) \rightarrow D_{s} \bar{D}_{s}^{*}$ & $2.5-3.3$ & $0.11_{-0.11}^{+0.23} \mathrm{GeV}^{-1}$ & $0.4_{-0.4}^{+1.7}$ \\
$Y(4660) \rightarrow \psi^{\prime} f_{0}(980) \rightarrow \psi^{\prime} \pi^{+} \pi^{-}$ & $4.4-5.4$ & $7.00_{-2.20}^{+2.24} \mathrm{GeV}^{+4.1}$ & $5.5_{-2.9}^{+4.1}$ \\
$Y(4660) \rightarrow J / \psi \phi(1020)$ & & $\sim 0$ & $\sim 0$ \\
\hline
\end{tabular}

$m_{Y}=4.66 \mathrm{GeV}$ and $\lambda_{Y}=6.74 \times 10^{-2} \mathrm{GeV}^{5}$. In this article, we choose a slightly smaller value $\lambda_{Y}=6.72 \times 10^{-2} \mathrm{GeV}^{5}$, which corresponds to $m_{Y}=4.65 \mathrm{GeV}$. For more literatures on the decay constants of the charmonium or bottomonium states, one can consult Ref. [48].

At the QCD side, we take the vacuum condensates to be the standard values $\langle\bar{q} q\rangle=-(0.24 \pm 0.01 \mathrm{GeV})^{3},\langle\bar{s} s\rangle=(0.8 \pm$ $0.1)\langle\bar{q} q\rangle,\left\langle\bar{s} g_{s} \sigma G s\right\rangle=m_{0}^{2}\langle\bar{s} s\rangle, m_{0}^{2}=(0.8 \pm 0.1) \mathrm{GeV}^{2}$ at the energy scale $\mu=1 \mathrm{GeV}$ [39-41,49], and take the $\overline{M S}$ masses $m_{c}\left(m_{c}\right)=(1.275 \pm 0.025) \mathrm{GeV}$ and $m_{s}(\mu=$ $2 \mathrm{GeV})=(0.095 \pm 0.005) \mathrm{GeV}$ from the Particle Data Group [11]. Moreover, we take into account the energy-scale dependence of the quark condensate, mixed quark condensate and $\overline{M S}$ masses from the renormalization group equation,

$$
\begin{aligned}
&\langle\bar{s} s\rangle(\mu)=\langle\bar{s} s\rangle(1 \mathrm{GeV})\left[\frac{\alpha_{s}(1 \mathrm{GeV})}{\alpha_{s}(\mu)}\right]^{\frac{12}{33-2 n_{f}}} \\
&\left\langle\bar{s} g_{s} \sigma G s\right\rangle(\mu)=\left\langle\bar{s} g_{s} \sigma G s\right\rangle(1 \mathrm{GeV})\left[\frac{\alpha_{s}(1 \mathrm{GeV})}{\alpha_{s}(\mu)}\right]^{\frac{2}{33-2 n_{f}}}, \\
& m_{c}(\mu)= m_{c}\left(m_{c}\right)\left[\frac{\alpha_{s}(\mu)}{\alpha_{s}\left(m_{c}\right)}\right]^{\frac{12}{33-2 n_{f}}}, \\
& m_{s}(\mu)= m_{s}(2 \mathrm{GeV})\left[\frac{\alpha_{s}(\mu)}{\alpha_{s}(2 \mathrm{GeV})}\right]^{\frac{12}{33-2 n_{f}}} \\
& \alpha_{s}(\mu)= \frac{1}{b_{0} t}\left[1-\frac{b_{1}}{b_{0}^{2}} \frac{\log t}{t}\right. \\
&\left.+\frac{b_{1}^{2}\left(\log ^{2} t-\log t-1\right)+b_{0} b_{2}}{b_{0}^{4} t^{2}}\right]
\end{aligned}
$$

where $t=\log \frac{\mu^{2}}{\Lambda^{2}}, b_{0}=\frac{33-2 n_{f}}{12 \pi}, b_{1}=\frac{153-19 n_{f}}{24 \pi^{2}}, b_{2}=$ $\frac{2857-\frac{5033}{9} n_{f}+\frac{325}{27} n_{f}^{2}}{128 \pi^{3}}, \Lambda=210 \mathrm{MeV}, 292 \mathrm{MeV}$ and $332 \mathrm{MeV}$ for the flavors $n_{f}=5,4$ and 3, respectively [11,50], and evolve all the input parameters to the optimal energy scale $\mu$ with $n_{f}=4$ to extract the hadronic coupling constants.

In the QCD sum rules for the mass of the $Y(4660)$, the optimal energy scale of the QCD spectral density is $\mu=2.9 \mathrm{GeV}$ [24], which is determined by the energy scale formula $\mu=\sqrt{M_{X / Y / Z}^{2}-\left(2 \mathbb{M}_{c}\right)^{2}}$ with the updated value of the effective $c$-quark mass $\mathbb{M}_{c}=1.82 \mathrm{GeV}$ [25]. In the present QCD sum rules, if we choose the energy scale $\mu=2.9 \mathrm{GeV}$, we obtain an energy scale as large as the masses of the $\eta_{c}$ and $J / \psi$ and much larger than the masses of the $D_{s}$ and $D_{s}^{*}$, it is a too large energy scale. In this article, we take the energy scales of the QCD spectral densities to be $\mu=\frac{m_{\eta_{c}}}{2}=1.5 \mathrm{GeV}$, which is acceptable for the mesons $D$ and $J / \psi[51]$. We set the Borel parameters to be $T_{1}^{2}=T_{2}^{2}=T^{2}$ for simplicity. The unknown parameters are chosen as $C_{X^{\prime} J / \psi}+C_{X^{\prime} f_{0}}=-0.012 \mathrm{GeV}^{8}, C_{X^{\prime} \eta_{c}}+$ $C_{X^{\prime} \phi}=0.0016 \mathrm{GeV}^{6}, C_{X^{\prime} \chi_{c 0}}+C_{X^{\prime} \phi}=0.0135 \mathrm{GeV}^{8}$, $C_{X^{\prime} D_{s}}+C_{X^{\prime} \bar{D}_{s}}=0.0038 \mathrm{GeV}^{7}, C_{X^{\prime} D_{s}^{*}}+C_{X^{\prime} \bar{D}_{s}^{*}}=$ $0.006 \mathrm{GeV}^{7}, C_{X^{\prime} D_{s}}+C_{X^{\prime} \bar{D}_{s}^{*}}=0.001 \mathrm{GeV}^{6}, C_{X^{\prime} \psi^{\prime}}+$ $\widetilde{C}_{X^{\prime} f_{0}}=-0.018 \mathrm{GeV}^{8}$ to obtain platforms in the Borel windows, which are shown in Table 3 explicitly. The Borel windows $T_{\max }^{2}-T_{\min }^{2}=1.0 \mathrm{GeV}^{2}$ for the charmonium decays and $T_{\max }^{2}-T_{\min }^{2}=0.8 \mathrm{GeV}^{2}$ for the open-charm decays, where the $T_{\text {max }}^{2}$ and $T_{\text {min }}^{2}$ denote the maximum and minimum of the Borel parameters, respectively. In the Borel widows, the platforms are flat enough, see the central values in Figs. 1 and 2 .

In Figs. 1 and 2, we plot the hadronic coupling constants $G_{Y B C}$ with variations of the Borel parameters $T^{2}$ at much larger intervals than the Borel windows. From the figures, we can see that there appear platforms in the Borel windows indeed. After taking into account the uncertainties of the input parameters, we obtain the hadronic coupling constants, which are shown explicitly in Table 3 . Now it is straightforward to calculate the partial decay widths of the $Y(4660) \rightarrow J / \psi f_{0}(980), \eta_{c} \phi(1020), \chi_{c 0} \phi(1020), D_{s} \bar{D}_{s}$, $D_{s}^{*} \bar{D}_{s}^{*}, D_{s} \bar{D}_{s}^{*}, D_{s}^{*} \bar{D}_{s}$ with formula,

$\Gamma(Y(4660) \rightarrow B C)=\frac{p\left(m_{Y}, m_{B}, m_{C}\right)}{24 \pi m_{Y}^{2}}\left|T^{2}\right|$,

where $p(a, b, c)=\frac{\sqrt{\left[a^{2}-(b+c)^{2}\right]\left[a^{2}-(b-c)^{2}\right]}}{2 a}$, the $T$ are the scattering amplitudes defined in Eq. (39), the numerical values of the partial decay widths are shown in Table 3. 

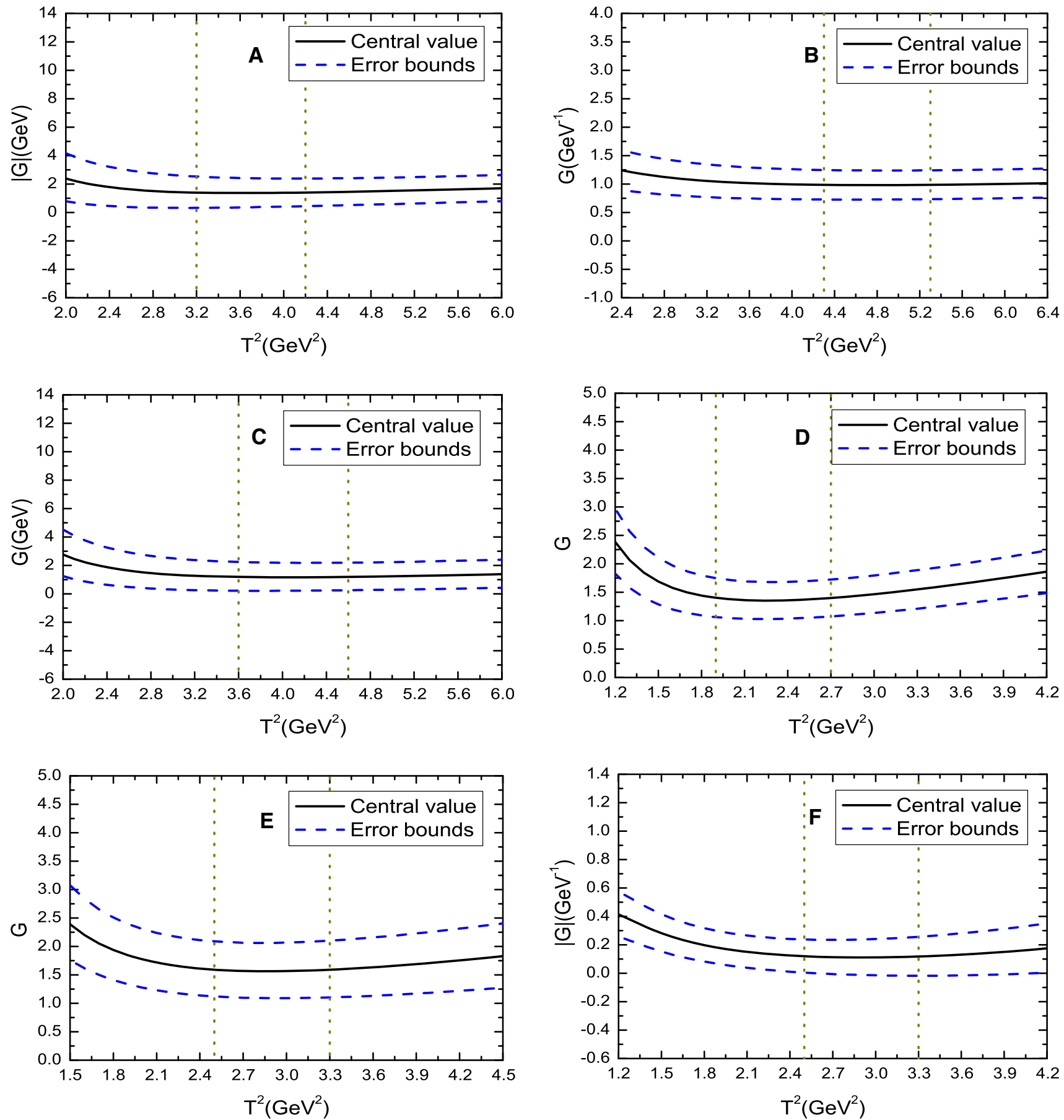

Fig. 1 The hadronic coupling constants with variations of the Borel parameters $T^{2}$, where the $A, B, C, D, E$ and $F$ denote the $G_{Y J} \psi f_{0}, G_{Y \eta_{c}} \phi$, $G_{Y \chi_{c 0} \phi}, G_{Y D_{s} \bar{D}_{s}}, G_{Y D_{s}^{*} \bar{D}_{s}^{*}}$ and $G_{Y D_{s} \bar{D}_{s}^{*}}$, respectively, the regions between the two perpendicular lines are the Borel windows

The decay $Y(4660) \rightarrow \psi^{\prime} f_{0}(980)$ is kinematically forbidden, but the decay $Y(4660) \rightarrow \psi^{\prime} \pi^{+} \pi^{-}$can take place through a virtual intermediate $f_{0}(980)^{*}$, the partial decay width can be written as,

$$
\begin{aligned}
\Gamma\left(Y \rightarrow \psi^{\prime} \pi^{+} \pi^{-}\right)= & \int_{4 m_{\pi}^{2}}^{\left(m_{Y}-m_{\psi^{\prime}}\right)^{2}} d s|T|^{2} \\
& \times \frac{p\left(m_{Y}, m_{\psi^{\prime}}, \sqrt{s}\right) p\left(\sqrt{s}, m_{\pi}, m_{\pi}\right)}{192 \pi^{3} m_{Y}^{2} \sqrt{s}}, \\
= & 5.5_{-2.9}^{+4.1} \mathrm{MeV},
\end{aligned}
$$




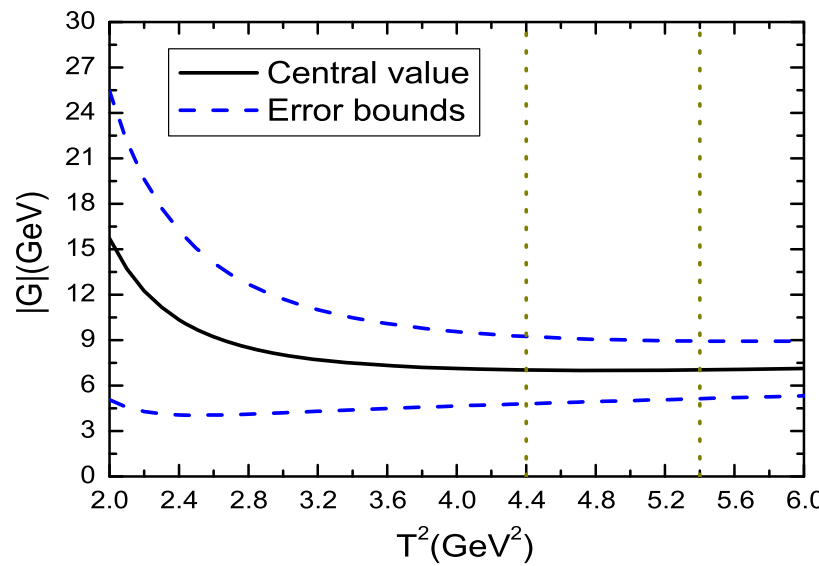

Fig. 2 The hadronic coupling constant $G_{Y \psi^{\prime} f_{0}}$ with variation of the Borel parameter $T^{2}$, the region between the two perpendicular lines is the Borel window

where

$$
\begin{aligned}
|T|^{2}= & \frac{\left(M_{Y}^{2}-s\right)^{2}+2\left(5 M_{Y}^{2}-s\right) M_{\psi^{\prime}}^{2}+M_{\psi^{\prime}}^{4}}{4 M_{Y}^{2} M_{\psi^{\prime}}^{2}} \\
& \times G_{Y \psi^{\prime} f_{0}}^{2} \frac{1}{\left(s-m_{f_{0}}^{2}\right)^{2}+s \Gamma_{f_{0}}^{2}(s)} G_{f_{0} \pi \pi}^{2}, \\
\Gamma_{f_{0}}(s)= & \Gamma_{f_{0}}\left(m_{f_{0}}^{2}\right) \frac{m_{f_{0}}^{2}}{s} \sqrt{\frac{s-4 m_{\pi}^{2}}{m_{f_{0}}^{2}-4 m_{\pi}^{2}}}, \\
\Gamma_{f_{0}}\left(m_{f_{0}}^{2}\right)= & \frac{G_{f_{0} \pi \pi}^{2}}{16 \pi m_{f_{0}}^{2}} \sqrt{m_{f_{0}}^{2}-4 m_{\pi}^{2}},
\end{aligned}
$$

$\Gamma_{f_{0}}\left(m_{f_{0}}^{2}\right)=50 \mathrm{MeV}$ [11], the hadronic coupling constant $G_{f_{0} \pi \pi}$ is defined by $\left\langle\pi^{+}(p) \pi^{-}(q) \mid f_{0}\left(p^{\prime}\right)\right\rangle=i G_{f_{0} \pi \pi}$.

Now it is easy to obtain the total decay width,

$\Gamma(Y(4660))=74.2_{-19.2}^{+29.2} \mathrm{MeV}$.

The predicted width $\Gamma(Y(4660))=74.2_{-19.2}^{+29.2} \mathrm{MeV}$ is in excellent agreement with the experimental data $68 \pm 11 \pm$ $1 \mathrm{MeV}$ from the Belle collaboration [3], which also supports assigning the $Y(4660)$ to be the $[s c]_{P}[\bar{s} \bar{c}]_{A}-[s c]_{A}[\bar{s} \bar{c}]_{P}$ type tetraquark state with $J^{P C}=1^{--}$.

From Table 3, we can see that the hadronic coupling constants $\left|G_{Y \psi^{\prime} f_{0}}\right|=7.00_{-2.20}^{+2.24} \mathrm{GeV} \gg\left|G_{Y J / \psi f_{0}}\right|=$ $1.37_{-1.04}^{+1.16} \mathrm{GeV}$, which indicates that the coupling

$Y(4660) \psi^{\prime} f_{0}(980)$ is very strong, and consistent with the observation of the $Y(4660)$ in the $\psi^{\prime} \pi^{+} \pi^{-}$mass spectrum, and favors the $\psi^{\prime} f_{0}(980)$ molecule assignment [12-15], as the strong coupling maybe lead to some $\psi^{\prime} f_{0}(980)$ component. Now we perform Fierz re-arrangement to the vector current $J_{\mu}(x)$ both in the color and Dirac-spinor spaces, and obtain the result,

$$
\begin{aligned}
J_{\mu}= & \frac{1}{2 \sqrt{2}}\left\{\bar{c} \gamma^{\mu} c \bar{s} s-\bar{c} c \bar{s} \gamma^{\mu} s+i \bar{c} \gamma^{\mu} \gamma_{5} s \bar{s} i \gamma_{5} c\right. \\
& -i \bar{c} i \gamma_{5} s \bar{s} \gamma^{\mu} \gamma_{5} c \\
& -i \bar{c} \gamma_{\nu} \gamma_{5} c \bar{s} \sigma^{\mu \nu} \gamma_{5} s+i \bar{c} \sigma^{\mu \nu} \gamma_{5} c \bar{s} \gamma_{\nu} \gamma_{5} s \\
& \left.-i \bar{s} \gamma_{\nu} c \bar{c} \sigma^{\mu \nu} s+i \bar{s} \sigma^{\mu \nu} c \bar{c} \gamma_{\nu} s\right\} .
\end{aligned}
$$

The $J_{\mu}(x)$ can be taken as a special superposition of color singlet-singlet type currents, which couple potentially to the meson-meson pairs or molecular states. The first term $\bar{c} \gamma^{\mu} c \bar{s} s$ is the molecule current chosen in Refs. $[14,15]$, which couples potentially to the $\psi^{\prime} f_{0}(980)$ molecular state. There does not exist a term $\bar{c} \sigma_{\mu \nu} c \bar{s} \gamma^{\nu} s$, which couples potentially to the $J / \psi \phi(1020)$ or $\psi^{\prime} \phi(1020)$ molecular state or scattering state. In calculations, we observe that the QCD side of the component $\Pi\left(p^{\prime 2}, p^{2}, q^{2}\right)$ in the correlation function $\Pi_{\alpha \beta v}(p, q)$ in Eq. (37) is zero at the leading order approximation, the hadronic coupling constant $G_{Y J / \psi \phi} \approx 0$. The decay $Y(4660) \rightarrow J / \psi \phi(1020)$ is greatly suppressed and can take place only through rescattering mechanism. It is important to search for the process $Y(4660) \rightarrow J / \psi \phi(1020)$ to diagnose the structure of the $Y(4660)$.

In Ref.[21], Sundu, Agaev and Azizi choose the $[s c]_{S}[\bar{s} \bar{c}]_{V}+[s c]_{V}[\bar{s} \bar{c}]_{S}$ type current to study the mass and width of the $Y(4660)$, and obtain the values $m_{Y}=$ $4677_{-63}^{+71} \mathrm{MeV}$ and $\Gamma_{Y}=(64.8 \pm 10.8) \mathrm{MeV}$ by saturating the width with the decays $Y(4660) \rightarrow J / \psi f_{0}(500)$, $J / \psi f_{0}(980), \psi^{\prime} f_{0}(500), \psi^{\prime} f_{0}(980)$. If the experimental value $m_{Y}=4652 \pm 10 \pm 8 \mathrm{MeV}$ is taken, the decay $Y(4660) \rightarrow \psi^{\prime} f_{0}(980)$ is kinematically forbidden, and can only take place through the upper tail of the mass distribution, the prediction $\Gamma\left(Y(4660) \rightarrow \psi^{\prime} f_{0}(980)\right)=30.2 \pm 8.5 \mathrm{MeV}$ is too large. Furthermore, other decay channels should be taken into account.

\section{Conclusion}

In this article, we illustrate how to calculate the hadronic coupling constants in the strong decays of the tetraquark states based on solid quark-hadron quality, then study the hadronic coupling constants $G_{Y J / \psi f_{0}}, G_{Y \eta_{c} \phi}, G_{Y \chi_{c 0} \phi}$, $G_{Y D_{s} \bar{D}_{s}}, G_{Y D_{s}^{*} \bar{D}_{s}^{*}}, G_{Y D_{s} \bar{D}_{s}^{*}}, G_{Y \psi^{\prime} f_{0}}, G_{Y J / \psi \phi}$ in the decays $Y(4660) \rightarrow J / \psi f_{0}(980), \eta_{c} \phi(1020), \chi_{c 0} \phi(1020), D_{s} \bar{D}_{s}$, $D_{s}^{*} \bar{D}_{s}^{*}, D_{s} \bar{D}_{s}^{*}, \psi^{\prime} \pi^{+} \pi^{-}, J / \psi \phi(1020)$ with the QCD sum rules in a systematic way. The predicted width $\Gamma(Y(4660))=$ $74.2_{-19.2}^{+29.2} \mathrm{MeV}$ is in excellent agreement with the experimental data $68 \pm 11 \pm 1 \mathrm{MeV}$ from the Belle collaboration, which supports assigning the $Y(4660)$ to be the $[s c]_{P}[\bar{s} \bar{c}]_{A}-[s c]_{A}[\bar{s} \bar{c}]_{P}$ type tetraquark state with $J^{P C}=$ $1^{--}$. In calculations, we observe that the hadronic coupling constants $\left|G_{Y \psi^{\prime} f_{0}}\right| \gg\left|G_{Y J / \psi f_{0}}\right|$, which indicates that the coupling $Y(4660) \psi^{\prime} f_{0}(980)$ is very strong, and consistent 
with the observation of the $Y(4660)$ in the $\psi^{\prime} \pi^{+} \pi^{-}$mass spectrum, and favors the $\psi^{\prime} f_{0}(980)$ molecule assignment, as there may be appear some $\psi^{\prime} f_{0}(980)$ component due to the strong coupling. The decay $Y(4660) \rightarrow J / \psi \phi(1020)$ is greatly suppressed and can take place only through rescattering mechanism. It is important to search for the process $Y(4660) \rightarrow J / \psi \phi(1020)$ to diagnose the nature of the $Y(4660)$.

Acknowledgements This work is supported by National Natural Science Foundation, Grant Number 11775079.

Data Availability Statement This manuscript has no associated data or the data will not be deposited. [Authors' comment: All data included in this manuscript are available upon request by contacting with the corresponding author.]

Open Access This article is distributed under the terms of the Creative Commons Attribution 4.0 International License (http://creativecomm ons.org/licenses/by/4.0/), which permits unrestricted use, distribution, and reproduction in any medium, provided you give appropriate credit to the original author(s) and the source, provide a link to the Creative Commons license, and indicate if changes were made. Funded by SCOAP ${ }^{3}$.

\section{References}

1. X.L. Wang et al., Phys. Rev. Lett. 99, 142002 (2007)

2. G. Pakhlova et al., Phys. Rev. Lett. 101, 172001 (2008)

3. X.L. Wang et al., Phys. Rev. D 91, 112007 (2015)

4. T. Barnes, S. Godfrey, E.S. Swanson, Phys. Rev. D 72, 054026 (2005)

5. W.J. Deng, H. Liu, L.C. Gui, X.H. Zhong, Phys. Rev. D 95, 034026 (2017)

6. B.Q. Li, K.T. Chao, Phys. Rev. D 79, 094004 (2009)

7. F.E. Close, N.A. Tornqvist, J. Phys. G 28, R249 (2002)

8. C. Amsler, N.A. Tornqvist, Phys. Rept. 389, 61 (2004)

9. L. Maiani, F. Piccinini, A.D. Polosa, V. Riquer, Phys. Rev. Lett. 93, $212002(2004)$

10. Z.G. Wang, Eur. Phys. J. C 76, 427 (2016)

11. M. Tanabashi et al., Phys. Rev. D 98, 030001 (2018)

12. F.K. Guo, C. Hanhart, U.G. Meissner, Phys. Lett. B 665, 26 (2008)

13. F.K. Guo, J. Haidenbauer, C. Hanhart, U.G. Meissner, Phys. Rev. D 82, 094008 (2010)
14. Z.G. Wang, X.H. Zhang, Commun. Theor. Phys. 54, 323 (2010)

15. R.M. Albuquerque, M. Nielsen, R. Rodrigues da Silva, Phys. Rev. D 84, 116004 (2011)

16. S. Dubynskiy, M.B. Voloshin, Phys. Lett. B 666, 344 (2008)

17. D. Ebert, R.N. Faustov, V.O. Galkin, Eur. Phys. J. C 58, 399 (2008)

18. G. Cotugno, R. Faccini, A.D. Polosa, C. Sabelli, Phys. Rev. Lett. 104, 132005 (2010)

19. L.Y. Xiao, X.Z. Weng, Q.F. Lu, X.H. Zhong, S.L. Zhu, Eur. Phys. J. C 78, 605 (2018)

20. R.M. Albuquerque, M. Nielsen, Nucl. Phys. A 815, 532009 (2009) [Erratum-ibid. A 857, 48 (2011)]

21. H. Sundu, S.S. Agaev, K. Azizi, Phys. Rev. D 98, 054021 (2018)

22. W. Chen, S.L. Zhu, Phys. Rev. D 83, 034010 (2011)

23. Z.G. Wang, Eur. Phys. J. C 74, 2874 (2014)

24. Z.G. Wang, Eur. Phys. J. C 78, 518 (2018)

25. Z.G. Wang, Eur. Phys. J. C 76, 387 (2016)

26. J.R. Zhang, M.Q. Huang, Phys. Rev. D 83, 036005 (2011)

27. Z.G. Wang, T. Huang, Nucl. Phys. A 930, 63 (2014)

28. Z.G. Wang, Commun. Theor. Phys. 63, 325 (2015)

29. Z.G. Wang, T. Huang, Eur. Phys. J. C 74, 2891 (2014)

30. Z.G. Wang, Eur. Phys. J. C 74, 2963 (2014)

31. Z.G. Wang, Eur. Phys. J. C 76, 70 (2016)

32. Z.G. Wang, T. Huang, Eur. Phys. J. C 76, 43 (2016)

33. Z.G. Wang, Nucl. Phys. B 913, 163 (2016)

34. Z.G. Wang, Eur. Phys. J. C 78, 933 (2018)

35. Z.G. Wang, Eur. Phys. J. C 79, 29 (2019)

36. Z.G. Wang, J.X. Zhang, Eur. Phys. J. C 78, 14 (2018)

37. Z.G. Wang, Z.Y. Di, Eur. Phys. J. C 79, 72 (2019)

38. Z.G. Wang. arXiv:1812.04503

39. M.A. Shifman, A.I. Vainshtein, V.I. Zakharov, Nucl. Phys. B 147, 385 (1979)

40. M.A. Shifman, A.I. Vainshtein, V.I. Zakharov, Nucl. Phys. B 147, 448 (1979)

41. L.J. Reinders, H. Rubinstein, S. Yazaki, Phys. Rept. 127, 1 (1985)

42. D. Becirevic, G. Duplancic, B. Klajn, B. Melic, F. Sanfilippo, Nucl. Phys. B 883, 306 (2014)

43. Z.G. Wang, Eur. Phys. J. C 77, 174 (2017)

44. Z.G. Wang, W.M. Yang, S.L. Wan, Eur. Phys. J. C 37, 223 (2004)

45. Z.G. Wang, W.M. Yang, S.L. Wan, Nucl. Phys. A 744, 156 (2004)

46. Z.G. Wang, Eur. Phys. J. C 75, 427 (2015)

47. V.A. Novikov, L.B. Okun, M.A. Shifman, A.I. Vainshtein, M.B. Voloshin, V.I. Zakharov, Phys. Rept. 41, 1 (1978)

48. Z.G. Wang, W.M. Yang, S.L. Wan, Phys. Lett. B 615, 79 (2005)

49. P. Colangelo, A. Khodjamirian. arXiv:hep-ph/0010175

50. S. Narison, R. Tarrach, Phys. Lett. 125B, 217 (1983)

51. Z.G. Wang, T. Huang, Phys. Rev. D 89, 054019 (2014) 